\title{
Adsorption behavior of organic molecules: a study of benzotriazole on Cu(111) with spectroscopic and theoretical methods
}

Article

Accepted Version

Gattinoni, C., Tsaousis, P., Euaruksakul, C., Price, R., Duncan, D. A., Pascal, T., Prendergast, D., Held, G. and Michaelides, A. (2019) Adsorption behavior of organic molecules: a study of benzotriazole on $\mathrm{Cu}(111)$ with spectroscopic and theoretical methods. Langmuir, 35 (4). pp. 882-893. ISSN 0743-7463 doi: https://doi.org/10.1021/acs.langmuir.8b03528 Available at https://centaur.reading.ac.uk/81701/

It is advisable to refer to the publisher's version if you intend to cite from the work. See Guidance on citing.

Published version at: http://dx.doi.org/10.1021/acs.langmuir.8b03528

To link to this article DOI: http://dx.doi.org/10.1021/acs.langmuir.8b03528

Publisher: ACS

All outputs in CentAUR are protected by Intellectual Property Rights law, including copyright law. Copyright and IPR is retained by the creators or other copyright holders. Terms and conditions for use of this material are defined in the End User Agreement. 


\section{CentAUR}

Central Archive at the University of Reading

Reading's research outputs online 


\section{Adsorption behavior of organic molecules: a study of benzotriazole on $\mathrm{Cu}(111)$ with spectroscopic and theoretical methods}

Chiara Gattinoni, ${ }^{\dagger}$ Panayiotis Tsaousis, ${ }^{\ddagger}$ Chanan Euaruksakul, ${ }^{\ddagger}$ Rachel Price, ${ }^{\ddagger}$ David A. Duncan, Tod Pascal, ${ }^{\S}$ David Prendergast, ${ }^{\S}$ Georg Held, ${ }^{*,+}$, and Angelos Michaelides*, $\dagger$

$\dagger$ Thomas Young Centre, London Centre for Nanotechnology and Department of Physics and Astronomy, University College London, Gower Street, WC1E 6BT, UK $\ddagger$ Department of Chemistry, University of Reading, Reading RG6 6AD, UK

\Diamond Light Source Harwell Science and Innovation Campus, Didcot OX11 OQX, UK $\S$ Molecular Foundry, Lawrence Berkeley National Laboratory, Berkeley, CA 94720, USA

E-mail: g.held@reading.ac.uk; angelos.michaelides@ucl.ac.uk 


\begin{abstract}
The adsorption of organic molecules on solid substrates is important to applications in fields such as catalysis, photovoltaics, corrosion inhibition, adhesion, and sensors. The molecular level description of the surface-molecule interaction and of the adsorption structures in these complex systems is crucial to understand their properties and function. Here we present the investigation of one such system, benzotriazole (BTAH) on single crystal $\mathrm{Cu}(111)$ in vacuum conditions. BTAH is the most widely used corrosion inhibitor for copper and thus a molecule of great industrial relevance. We show that the co-application of a wide range of spectroscopic techniques with theoretical methods provides unique insight in the description of the atomistic details of the adsorbed structures. Specifically, spectroscopic photoemission, absorption and standing wave experiments combined with ab initio computational modeling allowed us to identify that benzotriazole forms overlayers of intact BTAH when deposited at low temperature and it dissociates into BTA and $\mathrm{H}$ at room temperature and above. The dissociated molecule then forms complex structures of mixed chains and dimers of BTA bound to copper adatoms. Our work also reveals that copper adatoms at low concentrations, such as the theoretically predicted superstructures cannot be be resolved by means of current X-ray photoelectron spectroscopy (XPS) as the modelled $\mathrm{Cu} 2 \mathrm{p}$ spectra are practically indistinguishable from those for a $\mathrm{Cu}$ surface without adatoms. Overall this study significantly deepens understanding of BTAH on $\mathrm{Cu}$ - a system studied for more than 50 years - and it highlights the benefits of combining spectroscopic and computational methods in order to obtain a complete picture of a complex adsorption system.
\end{abstract}




\section{Introduction}

Complex organic molecules have many uses in industry and technology. Their interaction with crystalline surfaces is at the core of a large, and growing, number of applications. A few examples can be found in the fields of engineering, such as in corrosion protection ${ }^{1}$ or friction reduction, ${ }^{2}$ in energy conversion and storage, where molecule-surface interactions are central to, for example, dye-sensitized solar cells ${ }^{3}$ or in biology, where surface functionalization is needed for many novel biodiagnostics tools. ${ }^{4}$ In all cases, an in-depth knowledge of the interface layer between the molecule and the surface is required to understand how the technology itself works. This is however not always achieved, and this lack of understanding is often a barrier to the improvement of these technologies. In the present work we show how this issue can be addressed via a combination of a variety of spectroscopic techniques and density functional theory. In particular, we apply these methods to the example of benzotriazole adsorbed on a copper substrate.

Benzotriazole (BTAH, shown in Fig. 1) is the most widely used corrosion inhibitor for copper which, like most metals, is subject to tarnishing and corrosion when exposed to a humid atmosphere. ${ }^{5}$ It owes its popularity to its efficiency and it has been consistently used in industrial applications since the 1940s, when it was first synthesized. ${ }^{6}$ The reason behind its ability to stop copper from tarnishing and corroding is, however, still elusive despite the large amount of research which has been performed over the years to this aim (as reviewed in Ref. ${ }^{7}$ ). Early experimental studies by means of X-ray diffraction, ${ }^{8}$ electrochemical measurements ${ }^{9}$ and infra-red spectroscopy ${ }^{10}$ established that BTAH forms a passivating film by adsorbing on metal or oxidized copper surfaces. The detailed structure of this film and its nanoscale atomistic details have not been unequivocally established, partly because of the

insolubility of BTAH in aqueous and many organic solutions, which has precluded, until recently, detailed structural and chemical studies.

Recent advances in experimental and computational methods have made it possible to study the adsorption of molecules on surfaces in ultra-high vacuum, thus solving the solu- 


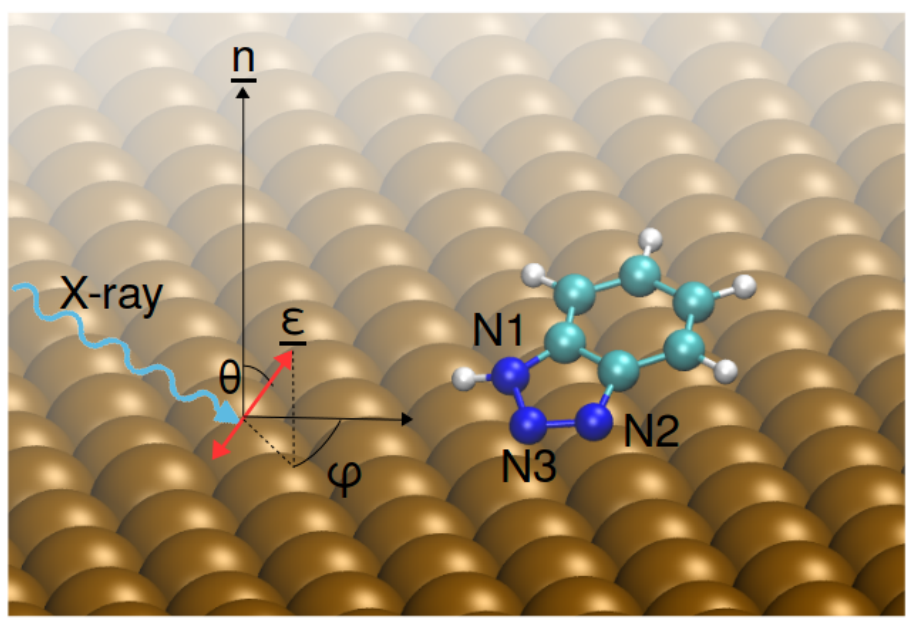

Figure 1: Representation of a BTAH molecule adsorbed on a $\mathrm{Cu}(111)$ surface. The BTA molecule differs from the BTAH by the loss of the hydrogen atom in the triazole moiety. On the left hand side the quantities used in Eq. 5 are defined: the surface normal $(\underline{n})$, the polarization of the X-ray $(\underline{\epsilon})$, the polar angle $(\theta)$ and the azimuthal angle $(\phi)$. Cu atoms are copper colored, carbons are cyan, nitrogens blue, and hydrogens while. This color scheme is used throughout.

bility issue and allowing us to focus on the nanoscale details of adsorption. In particular, synchrotron X-ray spectroscopy ${ }^{11}$ has revealed the adsorption structure of a wide range of molecules, from simple diatomic to complex ones, on many different substrates. Indeed, single-crystal spectroscopy under ultra-high vacuum (UHV) conditions can shine a light on the structure of a surface or an adsorbed film without interference from external atmospheric factors. At the same time, on the computational side, density functional theory (DFT) is the go-to tool for the study of adsorbed structures, where the accurate description of intermolecular and molecule-surface bonds is of paramount importance.

Benzotriazole is a complex organic molecule: it can chemisorb to the substrate via the azole moiety, physisorb via the benzene-like ring, ${ }^{12-15}$ form complex H-bonded overlayers when intact ${ }^{16,17}$ or instead deprotonate and form structures stabilized by copper adatoms. ${ }^{16,18-21}$ The application of some spectroscopic techniques has revealed that under UHV conditions BTAH dissociates into BTA and hydrogen, and that BTA forms ordered structures on the surface. ${ }^{8,22-27}$ The experimentally observed variety of structures includes 
flat lying chains, ${ }^{8,22,23}$ upright chains, ${ }^{24}$ upright isolated molecules ${ }^{25}$ and dimers. ${ }^{26,27}$ Also on the computational side, chain ${ }^{16,19-21}$ or dimer ${ }^{18}$ structures consisting of organometallic BTA-Cu complexes have been proposed.

It has been unclear so far whether these discrepancies arise from different experimental conditions, interpretation of the results or both. In the present work we show that the adsorption of such a complex organic molecule requires multiple experimental and computational techniques to uniquely identify its adsorbed structure and the application of one single method will likely lead to an incomplete description of the system. In particular, the interplay between theory and experiment is necessary in order to identify "hidden" features in the spectra, such as the coexistence of multiple structures or the presence of surface adatoms. Moreover, we highlight that when previous experimental results are re-interpreted in light of our findings good agreement is generally obtained between the different studies.

In the following, the orientation of the molecules with respect to the surface is investigated using near edge X-ray absorption fine structure (NEXAFS), chemical bonding using X-ray photoelectron spectroscopy (XPS) and NEXAFS, desorption and dissociation temperatures with temperature programmed XPS (TP-XPS) and distance of the molecule from the surface with normal-incidence X-ray standing wave (NIXSW). DFT is also used to complement and help interpret this suite of experimental techniques. Similar combinations of spectroscopic methods and DFT have been successfully employed to describe the structure of systems of adsorbed molecules on metals such as the interface between liquid water and gold, ${ }^{28}$ methyl acetoacetate and nickel, ${ }^{29,30}$ ethylene and $\mathrm{Si}(100)^{31}$ or a dye and $\mathrm{ZnO},{ }^{32}$ to name but a few. We restrict our work to $\mathrm{Cu}(111)$ as it is the lowest energy surface for copper and thus the dominant face exposed.

The structure of the paper is as follows: the Methodology section introduces the experimental and computational methods; this is followed by the Results, where we show first the temperature-dependent behavior of the adsorbed system, then the structure of the adsorbed layer at $300 \mathrm{~K}$ for two BTAH coverages and finally we investigate the structure of 
the $\mathrm{Cu}(111)$ surface and the presence of $\mathrm{Cu}$ adatoms. The Discussion and Conclusions are then presented.

\section{Methodology}

\section{Experimental}

The experiments were carried out at beamline I09 of the Diamond light source. The beamline features two canted undulators for soft X-rays (0.1 - $1.5 \mathrm{keV})$ and hard X-rays $(2.1$ - $15 \mathrm{keV})$, respectively. The endstation has a UHV analysis chamber with a base pressure in the low $10^{-10}$ mbar range. It is equipped with a Scienta EW4000 HAXPES hemispherical electron energy analyzer with an acceptance angle of $\pm 30^{\circ}$, which was used for X-ray photoelectron spectroscopy, near-edge X-ray absorption fine structure spectroscopy, and normal-incidence X-ray standing wave data collection. The analyzer was mounted at an angle of $90^{\circ}\left(83^{\circ}\right)$ with respect to the hard (soft) X-ray beam in the plane of the photon polarization.

The $\mathrm{Cu}$ single crystal was clamped onto a heater plate, which was mounted on a liquid nitrogen-cooled cold finger, thus allowing heating and cooling between $120 \mathrm{~K}$ and $900 \mathrm{~K}$. The $\mathrm{Cu}(111)$ surface was cleaned by repeated cycles of Ar sputtering at room temperature and annealing to $900 \mathrm{~K}$ until all contaminants' XPS signals were below the detection limit $(<0.01 \mathrm{ML})$. The benzotriazole layers were deposited prior to the spectroscopy experiments in a preparation chamber which can be separated from the analysis chamber and allows transfer in vacuum. Prior to dosing, benzotriazole (Sigma Aldrich, purity 99\%) was heated to $55^{\circ} \mathrm{C}$ in a pumped test tube and thoroughly degassed. At this temperature the vapor pressure is high enough to dose $100 \%$ saturation quantities directly onto the surface through a needle valve connected to the UHV chamber. Benzotriazole was dosed at pressures in the $10^{-8}$ mbar range.

XPS spectra in the $\mathrm{C} 1 \mathrm{~s}, \mathrm{~N} 1 \mathrm{~s}$ and $\mathrm{Cu} 2 \mathrm{p}$ regions were recorded at $10^{\circ}$ off-normal emission using photon energies of $420 \mathrm{eV}, 515 \mathrm{eV}$ and $1080 \mathrm{eV}$, respectively. The binding energies 
(BE) were calibrated with respect to the Fermi energy which was determined by measuring spectra of the Fermi edge using the same parameters (monochromator settings, pass energy, etc.). In addition, the spectra were normalized with respect to the low BE background. The overall energy resolution for the XPS data shown here is around $0.2 \mathrm{eV}$. Fast temperatureprogrammed (TP-)XPS spectra were recorded while the sample was annealed at a rate of $0.1 \mathrm{~K} \mathrm{~s}^{-1}$ (6.1 K per spectrum). C and $\mathrm{N} \mathrm{K}$-edge NEXAFS spectra were recorded using the KLL Auger electron yields integrated over the kinetic energy ranges 252.5 to 267.5 eV and 372.5 to $383.5 \mathrm{eV}$, respectively. Spectra of the clean surface were subtracted from those of the adsorbate-covered surfaces. The N K edge spectra were then divided by the beamline flux $\left(I_{0}\right)$ measured with a gold mesh inserted in the beamline. The C K-edge spectra were divided by the integrated signal of the Cu 3p XPS peak, measured with the same photon energy, as the $I_{0}$ signal showed features of carbon contamination. Even with this procedure, however, features due to contamination of the beamline optics could not be removed completely and/or discriminated from genuine adsorbate-induced features, especially in the pre-edge region. All spectra shown here were normalized at $320 \mathrm{eV}$ and $425 \mathrm{eV}$, respectively.

The normal incidence X-ray standing wave (NIXSW ${ }^{33}$ ) measurements were performed in the same endstation. The integrated intensity of the $\mathrm{N}$ 1s photoemission peaks were used to monitor the relative X-ray absorption of the $\mathrm{N}$ atoms. The photoemission spectra were acquired in fixed energy mode with a pass energy of $500 \mathrm{eV}$. A calibration curve was generated by dividing a fixed energy mode scan by a swept energy mode scan with the same step size and comparable acquisition time acquired over an area of the XP spectrum that was comparably flat. This calibration curve was used to normalize the $\mathrm{N}$ 1s photoemission spectra, which were then fitted with a linear background and four Gaussian line shapes corresponding to the two chemically unique $\mathrm{N}$ species in the adsorbed molecule (see below) and two shake-up energy loss features. Non-dipolar effects, due to the angular dependence of the photoemission, were modeled using theoretically calculated values, as described in Ref. ${ }^{34}$ and in the Supporting Information. The NIXSW measurements were repeated five times on 
different spots of the sample and the absorption profiles were fitted separately to produce an average value for the coherent fraction, $f_{\text {coh }}$, and coherent position, $p_{\text {coh }}$, and the associated random uncertainty. $f_{\text {coh }}$ and $p_{\text {coh }}$ are defined through the following equation:

$$
f_{\text {coh }} \cdot \exp \left(2 \pi i p_{\text {coh }}\right)=\sum_{n} f_{n} \cdot \exp \left(2 \pi i p_{n}\right)
$$

The sum runs over all absorber atoms contributing to the respective $\mathrm{N}$ 1s peak. $p_{n}$ are the positions of $\mathrm{N}$ atoms above the (111) scattering planes in modulo units of the separation between the (111) planes of $\mathrm{Cu}$ atoms $\left(d_{111}=2.08 \AA\right) ; f_{n}$ are relative coverages (corrected by a Debye-Waller factor to account for thermal deviations from the ideal positions) of these atoms. $^{33}$

\section{Simulation}

Simulated NEXAFS spectra and XPS core level shifts were calculated using DFT-based methods for the most stable $\mathrm{BTA}(\mathrm{H}) / \mathrm{Cu}(111)$ structures identified in previous publications. ${ }^{16,18,19}$ In addition, a new upright monomer configuration has also been considered (see Fig. 2a). A variety of four-layer-thick (unless otherwise stated) cell sizes were used. Most low coverage structures were modeled using a $4 \times 4 \mathrm{Cu}(111)$ slab, except for the dimers (chains) where a $2 \times 7(5 \times 4)$ system was used. For the high coverage case a $2 \times 4$ slab was employed for all structures except the chains, where a $2 \times 5$ one was used. All the structures have been optimized using VASP, ${ }^{35-38}$ with the optB86b-vdW functional ${ }^{39}$ and the projector augmented wave (PAW) method. ${ }^{40}$ The kinetic energy cutoff on the basis set was $400 \mathrm{eV}$ and Monkhorst-Pack grids of $3 \times 3 \times 1(2 \times 6 \times 1 / 6 \times 3 \times 1) k$-points were used for the $4 \times 4$ and $5 \times 4(2 \times 7 / 2 \times 4$ and $2 \times 5)$ cells. Periodic images were separated by $\sim 20 \AA$ vacuum and the bottom $\mathrm{Cu}$ layer coordinates were kept fixed at bulk values. Convergence of all the settings has been thoroughly checked. ${ }^{16}$ Adsorption at low coverage was modeled between 1/16 ML and 1/7 ML, at high coverage between $1 / 5$ and $1 / 4 \mathrm{ML}$, where $1 \mathrm{ML}$ refers to 1 
molecule per surface $\mathrm{Cu}$ atom. Adsorption energies, $\mathrm{E}_{\mathrm{ads}}$, in Fig. 2 are calculated as:

$$
\mathrm{E}_{\mathrm{ads}}^{\alpha}=\mathrm{E}_{\mathrm{system}}-N_{\mathrm{BTAH}} \mathrm{E}_{\mathrm{BTAH}}-\mathrm{E}_{\mathrm{slab}}-\alpha \Delta \mathrm{N}_{\mathrm{Cu}} g_{\mathrm{Cu}},
$$

where $\mathrm{E}_{\mathrm{BTAH}}$ and $\mathrm{E}_{\text {slab }}$ are, respectively, the total energies of the BTAH molecule in the gas phase and of the substrate (with $\mathrm{Cu}$ adatoms). $\mathrm{E}_{\text {system }}$ is the total energy of the whole system, consisting of BTAH adsorbed on the $\mathrm{Cu}$ slab, for an intact structure, and of BTA and $\mathrm{H}$ co-adsorbed on the substrate, for the deprotonated case. $\mathrm{N}_{\mathrm{Cu}}$ is the number of adatoms and $\mathrm{g}_{\mathrm{Cu}}$ the calculated formation energy. The parameter $\alpha$ can take the values 0 (the adatoms are present on the surface prior to adsorption) or 1 (all adatoms have to be formed from the copper bulk). A thorough examination of the cost of adatom formation and how that affects the stability of the systems is presented in the Supplementary Information (SI).

XPS core shifts for $\mathrm{N}$ 1s and $\mathrm{Cu} 2 \mathrm{p}$ were calculated with VASP in the final state approximation (screening by the valence electrons is included only) using a modified PAW method. ${ }^{41}$ The core electron binding energy is calculated as the difference between the total energies of the final (excited) state and the initial (ground) state:

$$
\mathrm{E}_{\mathrm{B}}=\mathrm{E}_{\mathrm{f}}-\mathrm{E}_{\mathrm{i}}
$$

The specific quantity reported herein is the binding energy shift, $\Delta \mathrm{E}_{\mathrm{B}}$, relative to a reference system:

$$
\Delta \mathrm{E}_{\mathrm{B}}=\mathrm{E}_{\mathrm{B}}^{\mathrm{sys}}-\mathrm{E}_{\mathrm{B}}^{\mathrm{ref}}
$$

where $\mathrm{E}_{\mathrm{B}}^{\mathrm{ref}}$ is the binding energy for an excited atom chosen as reference and $\mathrm{E}_{\mathrm{B}}^{\mathrm{sys}}$ for the excited atom of interest. The model XPS spectra were generated from the shifts by the superposition of Gaussian-Lorentzian peaks (Gaussian-Lorentzian products) with $50 \%$ mixing and full width at half maximum FWHM $0.95 \mathrm{eV}(\mathrm{N} \mathrm{1s})$ and $0.80 \mathrm{eV}\left(\mathrm{Cu} \mathrm{2} \mathrm{p}_{3 / 2}\right)$, as determined from fits to the experimental data. These were weighted with the number of atoms 
for each species.

The NEXAFS spectra for the nitrogen and carbon K-edges were simulated using the XCH method ${ }^{42}$ which has been custom-built into the Quantum-ESPRESSO package. ${ }^{43}$ The core-hole excitation is imposed by replacing the ground state pseudopotential with a core-excited one derived from a constrained electronic structure configuration (e.g. $1 s^{1} 2 s^{2} 2 p^{4}$ for nitrogen). The X-ray adsorption cross-section is calculated using Fermi's golden rule, whose transition matrix $(\underline{\boldsymbol{A}})$ elements are calculated within the projector-augmented wave approximation. ${ }^{44}$ The $\theta$-dependent spectra (where $\theta$ is the angle between the polarization direction and the surface normal, see Fig. 1) are finally obtained via a 2D isotropic average of the cross section:

$$
I(\theta)=\frac{1}{2 \pi} \int_{0}^{2 \pi} \hat{\boldsymbol{\epsilon}} \cdot \underline{\boldsymbol{A}} \cdot \hat{\boldsymbol{\epsilon}} d \phi=\frac{1}{2}(x x+y y) \sin ^{2}(\theta)+z z \cos ^{2}(\theta) .
$$

In the above, $\hat{\boldsymbol{\epsilon}}$ is the polarization vector, $\phi$ the polar angle, $\theta$ the azimuthal angle and $x x$, $y y, z z$ the diagonal elements of the transition matrix $\underline{\boldsymbol{A}}$. The directions are defined as the $x y$ plane being parallel to the surface and the $z$ axis perpendicular, and corresponding to $\theta=0$. Similar to the XPS calculations, the excitation energies obtained from this DFTbased method deviate significantly from the experimental values. In order to match the simulated spectra with the experimental ones, a shift is applied such that the first peak of the $\pi^{*}$ resonance corresponds to the first peak in the experimental data. Calculations were performed on the pre-optimized structures (with VASP, as described above) at the $\Gamma$ point and a generalized implementation ${ }^{45}$ of the Shirley $k$-space interpolation scheme ${ }^{46}$ is used for Brillouin zone sampling. The PBE exchange-correlation functional, ${ }^{47}$ Vanderbilt ultrasoft pseudopotentials ${ }^{48}$ and a plane-wave basis set with a kinetic energy cutoff of $25 \mathrm{Ry}(340 \mathrm{eV})$ were used. For comparison and validation XPS core shifts were calculated also with the XCH method. The shifts obtained with VASP and XCH presented the same trends and the maximum differences observed in $\Delta \mathrm{E}_{\mathrm{B}}$ were of the order of $50 \mathrm{meV}$. This highlights that the two methods used for the calculations in this study are compatible. 


\section{Results}

\section{Adsorption models for benzotriazole on $\mathrm{Cu}(111)$}

In this section we use DFT to examine the adsorption energies and spectroscopic features for possible adsorption geometries of benzotriazole on $\mathrm{Cu}(111)$. All the computed spectra reported herein are new. The structures shown in Figure 2 (and, with more details, in Fig. S1) are taken from a large pool of previously published candidate structures. ${ }^{16,18,19}$ Only the most stable structures were considered for this work, as explained in detail in the SI. Although it is expected that the molecule is deprotonated (i.e. BTA) at room temperature, we also consider BTAH structures. The most stable BTAH configurations are shown in Fig. 2e for low coverage and Fig. 2f,g for high coverage. For BTA, we only consider the monomer (Fig. 2a), chains (Fig. 2b) and dimers (Fig. 2c) for low coverage, and stacked chains (Fig. 2d) and dimers (Fig. 2c) at high coverage. Figure 2 also lists the respective adsorption energies, as well as calculated N K-edge NEXAFS and N 1s XPS spectra. C 1s

XPS spectra were not calculated, as the chemical environment of the carbon atoms is largely the same in all model structures, namely an aromatic ring not directly interacting with the surface. Therefore no significant differences are expected. 

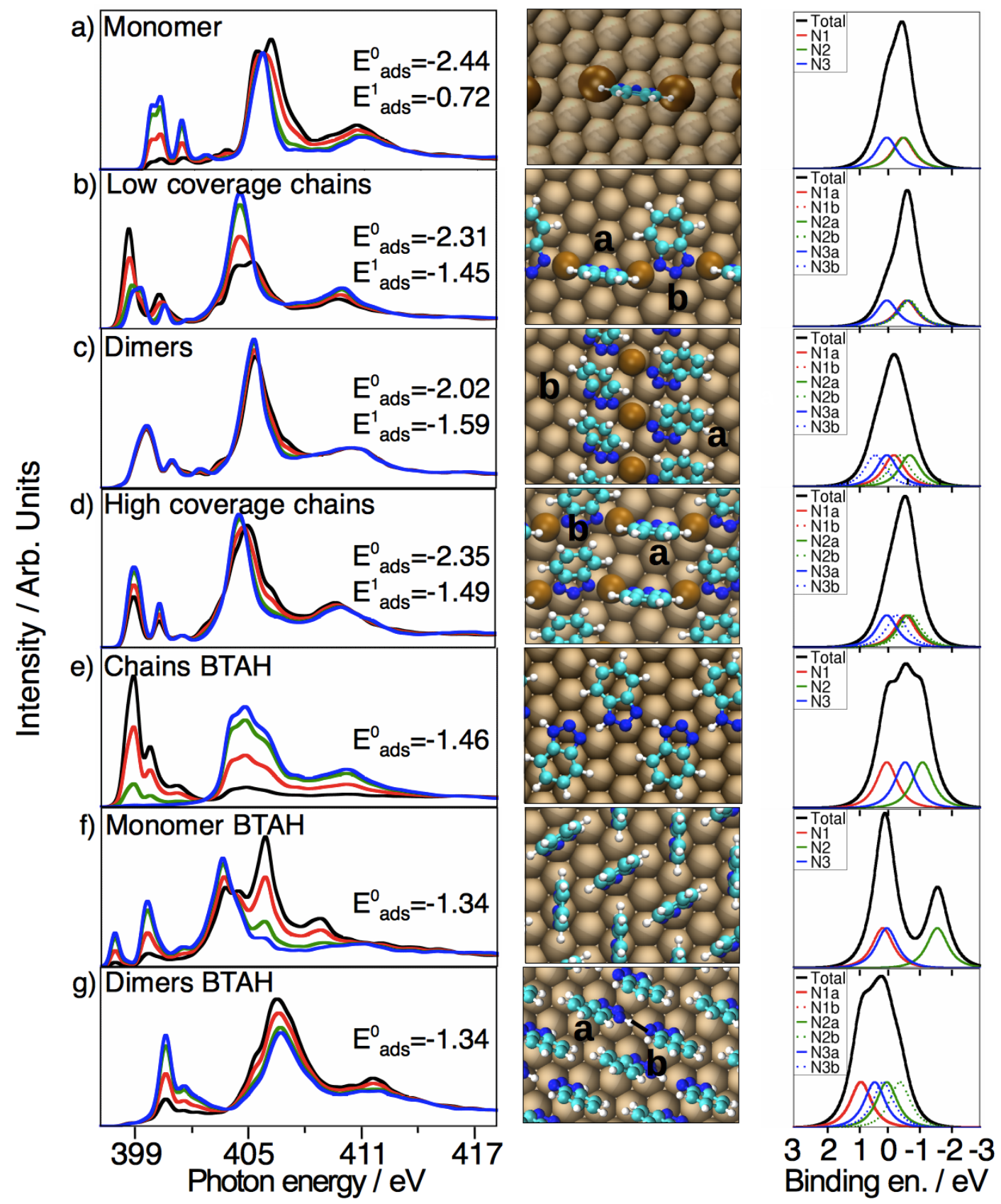

Figure 2: Simulated N K-edge NEXAFS (left) and simulated N 1s XPS (right) spectra for the BTA structures considered in this work, shown in the middle column. a) low coverage BTA monomer stabilized by two $\mathrm{Cu}_{\mathrm{ad}}$; b) low coverage BTA chains $\left(\frac{1}{10} \mathrm{ML}\right)$; c) high coverage BTA chains ( $\left.\frac{1}{5} \mathrm{ML}\right)$; d) BTA Dimers ( $\frac{1}{7} \mathrm{ML}$ for low coverage, $\frac{1}{4} \mathrm{ML}$ for high coverage); e) low coverage BTAH $\left.\left(\frac{1}{16} \mathrm{ML}\right) ; \mathrm{f}\right)$ high coverage BTAH dimers $\left(\frac{1}{4} \mathrm{ML}\right)$; g) high coverage BTAH monomers $\left(\frac{1}{4} \mathrm{ML}\right)$. The dark brown atoms on top of the $\mathrm{Cu}(111)$ surface (depicted in light brown) are copper adatoms. The NEXAFS spectra have been calculated for $\theta=17$ (black), 42 (red), 67 (blue), $87^{\circ}$ (green), where $\theta$ is the angle between the polarization and the normal to the surface. In the structural figures (middle panels), the $a$ and $b$ labels indicate molecules $a$ and $b$ in the corresponding XPS spectra. N1, N2 and N3 are defined in Fig. 1. In the XPS spectra the $\mathrm{N}$ atom closer to the surface has its calculated spectrum centered at 0 . Adsorption energies are calculated according to Eq. 2. 
The most stable adsorption structures are those of BTA in the presence of surface copper adatoms $\left(\mathrm{Cu}_{\mathrm{ad}}\right)$ acting as a link between neighboring BTAs (Fig. 2b-d) or stabilizing the BTA on the surface (Fig. 2a). In general, the presence of copper adatoms allows a larger number of strong $\mathrm{Cu}-\mathrm{N}$ bonds to be formed by the $\mathrm{N}$ atoms in the triazole moiety, whereby one nitrogen binds to a $\mathrm{Cu}$ atom within the surface layer and one (BTA dimer) or both (BTA monomer, chains) other nitrogens form bonds with the $\mathrm{Cu}$ adatoms. Structures of BTAs adsorbed on $\mathrm{Cu}(111)$ without $\mathrm{Cu}$ adatoms had been investigated in Ref. ${ }^{16,21}$ where they were found to be unfavorable with respect to the intact structures presented in Fig. 2. They are therefore not considered in this study. The structures of the low (Fig. 2b) and high-coverage (Fig. 2d) BTA chains both consist of alternating upright and tilted molecules forming three $\mathrm{N}-\mathrm{Cu}$ bonds each. The tilting of every second BTA in the chain structure is coverage-dependent, being almost flat at low coverage and $\sim 60^{\circ}$ with respect to the surface plane at high coverage. The BTA-Cu $u_{a d}-\mathrm{BTA}$ dimers (Fig. 2c) consist of nearly upright molecules tilted $\left(\sim 60^{\circ}\right.$ with respect to the surface) each forming two $\mathrm{N}-\mathrm{Cu}$ bonds, one with a $\mathrm{Cu}$ atom within the surface plane and one with an adatom. The dimers have the same structure at low and high coverage, the only difference being the lateral spacing between neighboring chains (therefore only one structure is shown in Fig. 2c). Because of the similar chemical environment of all three nitrogen atoms, DFT predicts only small chemical shifts of less than $1 \mathrm{eV}$ in the $\mathrm{N}$ 1s XP-spectra for all of the BTA structures. In the chains, at both coverages, only the central N3 atoms (the definition of N1, N2 and N3 is shown in Fig. 1) of both triazoles show a significant shift as they are the only $\mathrm{N}$ not binding strongly to a $\mathrm{Cu}$ adatom (Fig. 2b,d). The computed shift in binding energy for these $\mathrm{N} 3$ atoms is however only $0.64 \mathrm{eV}$ (at low coverage) and $0.58 \mathrm{eV}$ (at high coverage) higher with respect to the other nitrogens. For the dimers, the binding energies are equally spaced out for the six nitrogens examined, the range of binding energies being $0.8 \mathrm{eV}$.

The simulated N K-edge NEXAFS spectra for the BTA structures show two absorption peaks about $1.5 \mathrm{eV}$ apart, which are both associated with the $\pi^{*}$ system. The more intense 
peak at low photon energy is narrow for the chain structures (Fig. 2b,d), broader for the dimer (Fig. 2c) and split for the monomer structures (Fig. 2a). This reflects the different levels of $\mathrm{N}-\mathrm{Cu}$ bonding in these structures. The $\sigma^{*}$ system shows two distinct peaks at about 6 and $12 \mathrm{eV}$ above the first $\pi^{*}$-resonance. The angular dependence of both $\pi^{*}$ and $\sigma^{*}$ resonances is as expected for a system dominated by resonant $\mathrm{N}=\mathrm{N}$ and $\mathrm{N}=\mathrm{C}$ double-bonds with the respective orientations. ${ }^{49}$ The upright monomer structure (Fig. 2a) is characterized by a strong angular dependence in the $\pi^{*}$-resonance with the highest cross section close to normal incidence $\left(\theta=90^{\circ}\right)$. One component of the first $\sigma^{*}$-resonance peak shows the opposite behavior, whereas the other features are largely unaffected by the changes in the polarization vector. The angular dependence expected for the low-coverage chain structure (Fig. 2b) is almost opposite to that of the monomer with the highest absorption cross section for the main $\pi^{*}$-resonance peak at grazing incidence $\left(\theta=17^{\circ}\right)$. The cross section does not go to zero for normal incidence and instead a shoulder grows, which is due to the fact that the structure consists of two molecules with different orientations. Both $\sigma^{*}$-resonance peaks show the opposite angular behavior. The high-coverage chain structure (Fig. 2d) leads to a more moderate angular dependence as the tilt of one of the two molecules in the structure is close to the "magic angle" of $54.7^{\circ}$, for which no angular dependence is expected. For the same reason, the dimer structure (Fig. 2c) shows almost no angular dependence in the modeled NEXAFS spectra.

The Boltzmann probability distribution for these structures (shown in Fig. S2 of the Supporting Information) shows that every one of them is favorable for different conditions of the surface (i.e. whether the surface is more or less disordered with many or few adatoms) and therefore they all have to be carefully considered.

The character of the adsorption structures for non-deprotonated BTAH (Fig. 2e-g) changes according to the coverage. At low coverage $\left(\frac{1}{16} \mathrm{ML}\right)$ the most stable structures consist of H-bonded chains with the BTAHs lying almost parallel to the surface (Fig. 2e). At high coverage $\left(\frac{1}{4} \mathrm{ML}\right)$ all the BTAHs are upright, and their adsorption is stabilized by the forma- 
tion of $\mathrm{N}-\mathrm{Cu}$ bonds. Two isoenergetic structures are observed: a monomer structure with no hydrogen bonds (Fig. 2f) and a H-bonded dimer configuration (Fig. 2g). Structures with higher coverages are unstable. Therefore, $\frac{1}{4} \mathrm{ML}$ can be considered as the saturation coverage for BTAH. As the protonated nitrogen atom has a different chemical environment from the other $\mathrm{N}$ atoms, larger chemical shifts are predicted for the BTAH structures, compared to BTA, and this is shown in the broader XPS spectra in Fig. 2e-g. In the low coverage chains the $\mathrm{N} 1$ atoms (which are part of the N-H in the triazole, see Fig. 1) have the highest binding energy and the $\mathrm{N} 2$ ones (which form an H-bond with the neighboring BTAH) the lowest. Their calculated binding energies are $\sim 0.6 \mathrm{eV}$ either side of the central N3 atom and 1.15 $\mathrm{eV}$ apart. The monomer (Fig. 2f) shows two distinct peaks almost $2 \mathrm{eV}$ apart: one for $\mathrm{N} 1$ and N3, and a second for $\mathrm{N} 2$ which is neither bonded to the triazole $\mathrm{H}$ (N1) nor to the surface (N3). Finally, in the dimers (Fig. 2g) the peaks corresponding to the six $\mathrm{N}$ atoms of the structure are fairly spread apart over $1.5 \mathrm{eV}$, with the $\mathrm{N} 1$ atoms having the highest binding energies.

The simulated N K-edge NEXAFS spectra for BTAH at low coverage (Fig. 2e) show a $\pi^{*}$ system composed of three distinct peaks $\sim 1.5 \mathrm{eV}$ apart, which reflect the three chemical environments of the triazole nitrogen atoms: one involved in a H-bond, the central one in a $\mathrm{N}-\mathrm{Cu}$ bond and one bonded to a $\mathrm{H}$ atom. The $\sigma^{*}$ system shows two distinct peaks with opposite angular dependence with respect to the $\pi^{*}$ resonance i.e. the highest intensities are for grazing incidence in the $\pi^{*}$ resonance and normal for the $\sigma^{*}$. At high coverage, the spectra for the monomer (Fig. 2f) show two $\pi^{*}$ peaks of similar intensity spaced $\sim 1 \mathrm{eV}$ apart, and a $\sigma^{*}$ system with two peaks and a node. In the case of the dimer (Fig. 2g), there is instead a single peak with a shoulder for $\pi^{*}$ and two distinct peaks for $\sigma^{*}$. In both spectra the highest intensity for the $\pi^{*}$ resonance is at normal incidence and for the $\sigma^{*}$ at grazing angles. 

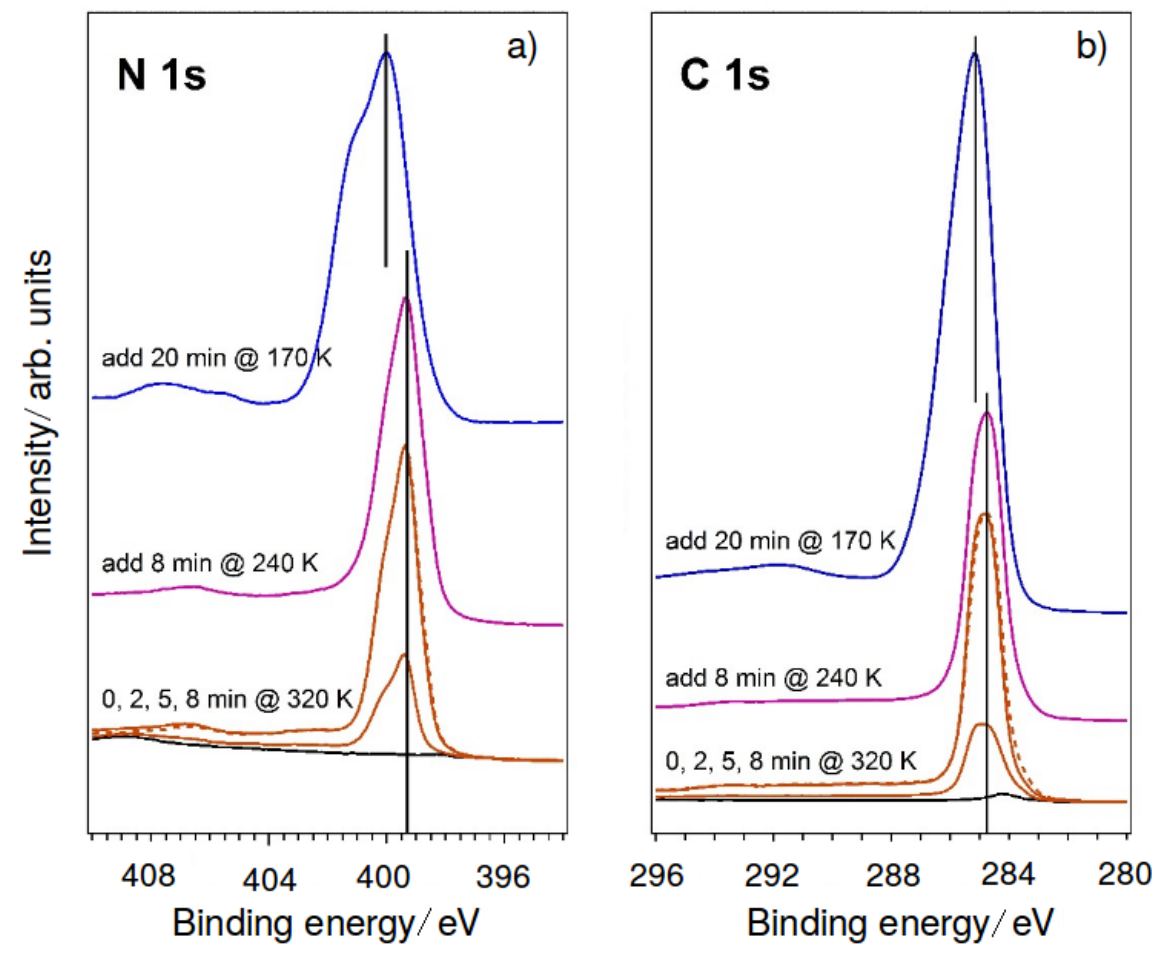

Figure 3: Measured XPS spectra for different levels of benzotriazole uptake and temperature. (a) $\mathrm{N} 1 \mathrm{~s}$ (photon energy $515 \mathrm{eV}$ ) (b) C 1s (photon energy $420 \mathrm{eV}$ ). The spectra at the bottom of the figure (black line) are for a nominally clean surface. The red lines correspond to dosing times of $2 \mathrm{~min}, 5 \mathrm{~min}$ (solid) and $8 \mathrm{~min}$ (dashed) at $320 \mathrm{~K}$; the purple spectrum in the middle corresponds to an additional $8 \mathrm{~min}$ dosing at $240 \mathrm{~K}$; the blue spectrum at the top corresponds to an additional $20 \mathrm{~min}$ at $170 \mathrm{~K}$.

\section{Temperature-dependent behavior of the adsorbed system}

The temperature dependence of the benzotriazole layer on $\mathrm{Cu}(111)$ was studied by means of XPS and TP-XPS. In particular, the onset of deprotonation into BTA and H, desorption and decomposition were investigated. When dosing benzotriazole at $320 \mathrm{~K}$, the intensities of the $\mathrm{C} 1 \mathrm{~s}$ and $\mathrm{N}$ 1s XPS spectra saturate after $5 \mathrm{~min}$ (Fig. 3). Indeed, the 8 min spectrum (dashed red line) and the 5 min one (solid red line) are identical. We define this layer as the saturated chemisorbed layer and use the relative intensities of the respective $\mathrm{N}$ 1s signals for 
coverage calibration of all other BTA layers studied in this work. Comparison of both the C 1s and $\mathrm{N}$ 1s peak intensities consistently leads to a coverage of $30 \%$ saturation for the 2 min spectrum. The $\mathrm{C}$ 1s spectra at $320 \mathrm{~K}$ show a single peak at BE $284.8 \mathrm{eV}$, which is consistent with carbon atoms in an organic system. The $\mathrm{N}$ 1s signal has a main peak at $399.4 \mathrm{eV}$, a shoulder at $400.3 \mathrm{eV}$, which is more pronounced for the low-coverage spectrum (2 min) than for saturation coverage, and a satellite feature around $407 \mathrm{eV}$. Additional dosing at $240 \mathrm{~K}$ does not increase the coverage significantly except for a small additional asymmetry at the high binding energy side of the main signal. Dosing at $170 \mathrm{~K}$, however, leads to multilayer growth and the main peaks of the spectra shift by about $0.5 \mathrm{eV}$ with respect to the chemisorbed species. After dosing for $20 \mathrm{~min}$ at $170 \mathrm{~K}$ the $\mathrm{Cu} 2 \mathrm{p}$ signal is completely attenuated ( $<1 \%$ of clean surface signal, not shown). Using the inelastic mean free path for $150 \mathrm{eV}$ electrons through glassy $\mathrm{C}(7.5 \AA)^{50}$ we estimate that the layer is at least $35 \AA$ thick.

Figure 4 shows N 1s spectra recorded for $36 \%$ and $100 \%$ saturated layers at $300 \mathrm{~K}$. We find good qualitative agreement between the experimental XPS spectra and the model spectra for low and high coverage BTA chains or dimers which are also shown in Fig. 4. The experimental spectra are broader, however they contain the same features as the calculated ones, i.e a main peak with a shoulder at high BE, which is more pronounced for the low coverage than for $100 \%$ saturation coverage. The narrow distribution of $\mathrm{N}$ 1s binding energies of less than $1 \mathrm{eV}$ in the experimental spectra clearly rules out the presence of BTAH on the surface, which would lead to broad or multiple features spread over $2-3 \mathrm{eV}$ (see Fig. 2 and 4 ).

TP-XPS reveals further insights into the thermal evolution of the benzotriazole layer. After dosing the equivalent of $114 \%$ of the saturated chemisorbed layer onto the clean surface at $280 \mathrm{~K}$ (Fig. 5b) very significant differences are observed in the $\mathrm{N}$ 1s signal compared to the layer dosed at $320 \mathrm{~K}$ (Fig. 3a) which are lifted once the sample is annealed to $320 \mathrm{~K}$ (Fig. 5a, b). These observations are explained by intact adsorption of benzotriazole (BTAH) 


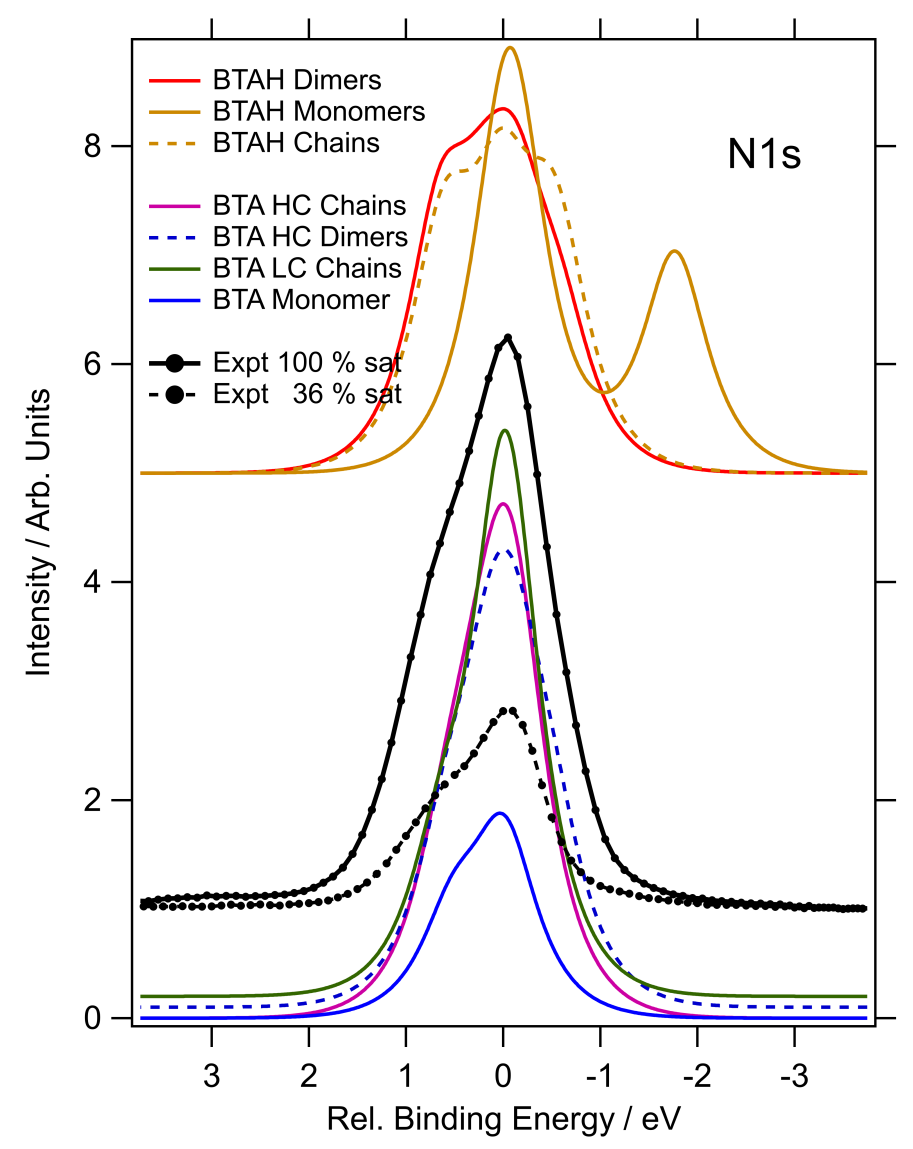

Figure 4: Comparison of experimental (100\% and $36 \%$ saturation) and computational N 1s XPS spectra. Experimental data are shown as dots with lines. Calculated spectra are shown as solid and dashed lines. In all the spectra the 0 of the relative binding energy corresponds to the position of the peak with the highest intensity.

at $280 \mathrm{~K}$ and reordering of the layer and dissociation into BTA and $\mathrm{H}$ upon annealing. Three distinct peaks at 399.2, 400.2, and $401.3 \mathrm{eV}$ are visible in the $281 \mathrm{~K}$ spectrum of Fig. $5 \mathrm{~b}$. Our model calculations show that this is a signature of high-coverage BTAH (see Figure 4). At $320 \mathrm{~K}$ the spectrum shows a single peak with a shoulder, as described above, which is in good agreement with theoretical predictions for deprotonated BTA (see Figure 4). The specific BTA structure formed at room temperature at high coverage will be discussed in the following Section. Between $320 \mathrm{~K}$ and $430 \mathrm{~K}$ the signal changes very little reflecting the stability of the surface layer, before desorption and decomposition of the molecules sets in above $430 \mathrm{~K}$ (as previously observed ${ }^{51}$ ). Between $430 \mathrm{~K}$ and $670 \mathrm{~K}$ the signals of intact BTA at $\mathrm{BE} 399.3 \mathrm{eV}$ and a decomposition feature with a $\mathrm{N}$ 1s peak at $398.0 \mathrm{eV}$ co-exist. The 


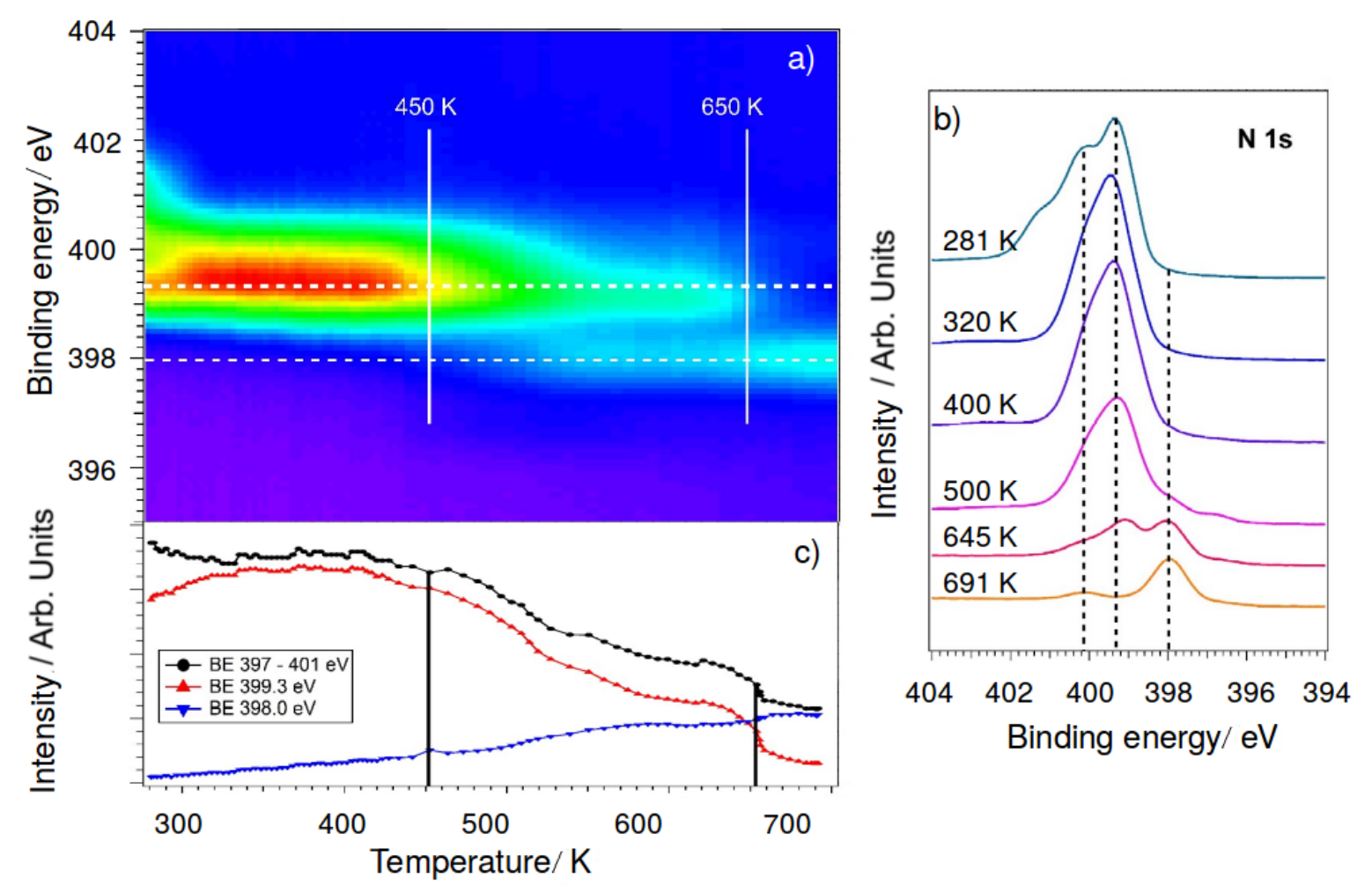

Figure 5: a) 2D plot showing the temperature dependence of the experimentally measured XPS signal.b) Single measured XPS spectra for a range of temperatures between $281 \mathrm{~K}$ and $691 \mathrm{~K} . \mathrm{c}$ ) Temperature dependence of the total N 1s signal between 397 and $401 \mathrm{eV}$ (black line), the signal at $399.3 \pm 0.4 \mathrm{eV}$ (intact molecule, red line) and $398.0 \pm 0.4 \mathrm{eV}$ (decomposition product, blue line).

temperature dependence of the total $\mathrm{N}$ 1s signal (see Fig. 5c) shows the signal of the intact BTA molecule decreasing by $87 \%$ over this temperature range, whereby $24 \%$ of the signal is lost in the final desorption step between $640 \mathrm{~K}$ and $670 \mathrm{~K}$. After annealing to $670 \mathrm{~K}$ the intensity of the decomposition peak at $398.0 \mathrm{eV}$ is only about $30 \%$ of the of the $\mathrm{N} 1 \mathrm{~s}$ signal of BTA at $320 \mathrm{~K}(\mathrm{BE} 399.3 \mathrm{eV})$. At the same time the total $\mathrm{N}$ 1s signal decreases by $60 \%$, i.e. about $60 \%$ of the molecules desorb whereas $40 \%$ decompose and their $\mathrm{N}$ atoms remain on the surface.

It has been previously reported ${ }^{52}$ that benzotriazole can reduce the rate of oxidation of 
copper in air up to a temperature of $\sim 620 \mathrm{~K}$. This is consistent with these results showing that benzotriazole or its dissociation products cover the surface up to this temperature.

\section{Adsorption at $300 \mathrm{~K}$}

\section{Deprotonation}

The XPS spectra discussed in the previous Section show that benzotriazole is deprotonated at room temperature. To add support to this conclusion, the structure of benzotriazole deposited at $50 \%$ and $100 \%$ saturation on $\mathrm{Cu}(111)$ was studied with NEXAFS, both for the $\mathrm{N}$ and the C K-edge. The combination of the experimental measurements and their comparison to DFT-optimized structures and calculated spectra allows us to conclude that the adsorbed overlayer consists of deprotonated BTA for both coverages. Indeed, the experimental N K-edge spectra show $\pi^{*}$-resonances at excitation energies between $398-401 \mathrm{eV}$ and $\sigma^{*}$ resonances at energies above $402 \mathrm{eV}$ (Fig. 6a, 8a). For both coverages, for the $\pi^{*}$-resonance, two clearly resolved peaks are observed and the $\sigma^{*}$ system has two features about $5 \mathrm{eV}$ apart. As the calculated spectra for BTAH predict a third peak in the $\pi^{*}$ system (chains, Fig. 2e) or a very different character in the $\sigma^{*}$ system (monomer and dimers, Fig. $2 \mathrm{f}$ and $\mathrm{g}$ ), this indicates that at $300 \mathrm{~K}$ benzotriazole is deprotonated into BTA and $\mathrm{H}$.

Model C K-edge spectra for all candidate structures are shown in Fig. S2 of the Supporting Information. They all show two equally intense $\pi^{*}$-resonance peaks (except for the BTAH monomer, where one main peak is observed instead), 1.2-1.7 eV apart. The lower excitation energy is associated with $\pi$ states within the $\mathrm{C}_{6}$-ring; the higher energy peak is an excitation in $\pi$-states near the $\mathrm{C}=\mathrm{N}$ bonds. The experimental $\mathrm{C} \mathrm{K}$-edge spectra recorded at $300 \mathrm{~K}$ for both $50 \%$ and $100 \%$ saturation coverage show two $\pi^{*}$-resonance peaks (Fig. 6b, 8b, solid lines), which is in good agreement with most of the model spectra, dissociated and intact. Therefore the $\mathrm{C}$ K-edge does not provide information regarding dissociation, which is expected as the loss of the $\mathrm{H}$ atom does not significantly affect the chemical environment of the benzene-like ring. 


\section{Structure at low coverage}

NEXAFS spectra for the $\mathrm{N}$ and $\mathrm{C}$ K-edge $\left(\theta=17^{\circ}, 42^{\circ}, 67^{\circ}\right)$ were collected for benzotriazole deposited at 50\% saturation and they are shown as solid lines in Fig. 6. These were compared

a) N K-edge

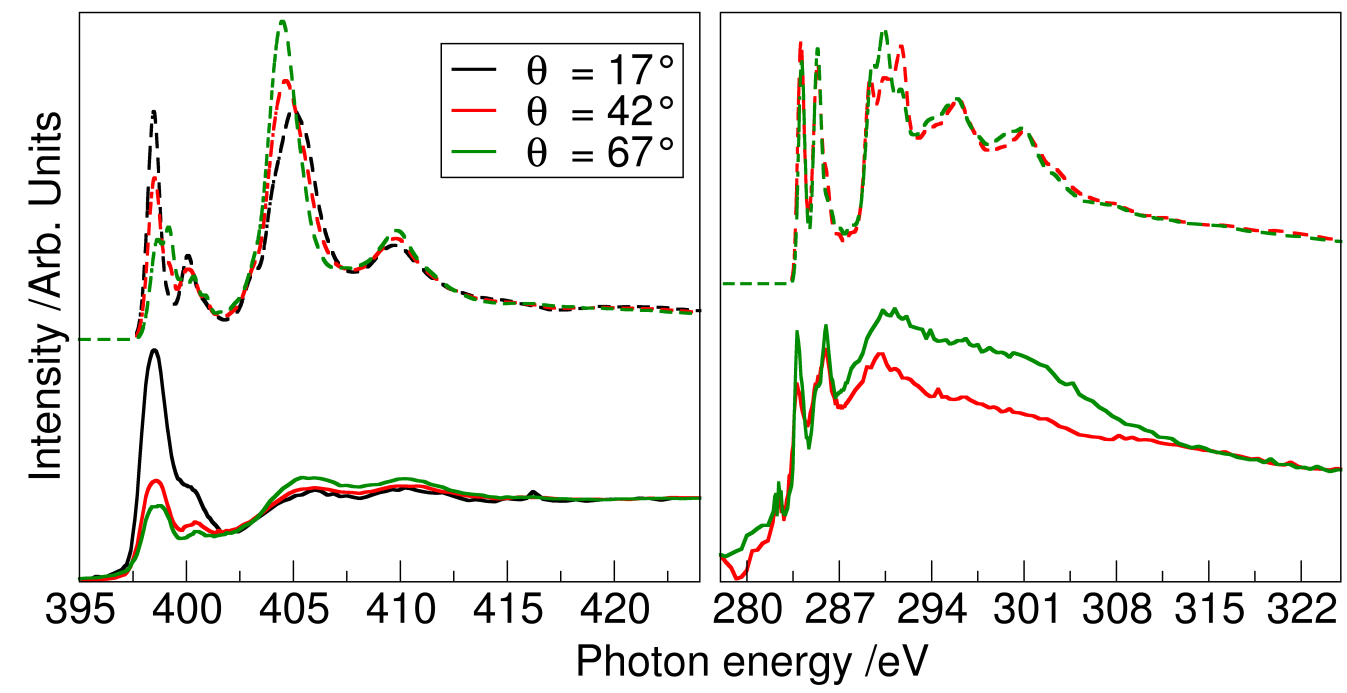

Figure 6: Comparison of experimental and computational spectra for the a) N K-edge and b) C K-edge for low coverage BTA adsorption at $300 \mathrm{~K}$. Experimental data are shown as solid lines. The computational spectra (dashed lines) are those for a mixture of $25 \%$ upright monomers (Fig. 2a) and $75 \%$ low coverage chains (Fig. 2b). The angle $\theta$ refers to the angle between the X-ray polarization vector and the surface normal (see Fig. 1).

to theoretical spectra for all structures described in the previous Section and shown in Fig. 2 and the best fit is shown as dashed lines in Fig. 6 .

The highest intensity for the $\mathrm{N} \mathrm{K}$-edge (Fig. 6a) is observed for $\theta=17^{\circ}$, indicating that there is a significant number of flat-lying molecules present at the surface. The low-coverage chain structure in Fig. 2b is the only deprotonated case where the calculated NEXAFS spectra show the highest $\pi^{*}$ cross section for low values of $\theta$. The low coverage chains alone provide good agreement with the experimental data. 
However, the C K-edge spectra suggest a that mixed adsorption structure of chains and upright monomers (up to 30\%) might be a better fit. Indeed, the angular dependence of the $\pi^{*}$ region in the experimental $\mathrm{C}$ spectra (Fig. 6b, solid lines) are more in line with an upright orientation of the $\mathrm{C}_{6}$-ring, contrary to the results obtained from the $\mathrm{N}$ K-edge. None of the theoretical spectra showed opposite angular dependence between $\mathrm{N}$ and $\mathrm{C}$ K-edge, as expected, since the $\pi^{*}$ orbitals are perpendicular to the plane of the molecule in both cases. In addition, one would expect that at least some of the $\sigma^{*}$-resonance show an opposite behavior to the $\pi^{*}$-resonance, which is not observed in these experimental spectra. Compared to the $\mathrm{N}$ K-edge, the data collection of $\mathrm{C}$ K-edge NEXAFS spectra is more challenging because carbon contamination of the beamline optical elements results in very significant drops in the beamline transmission in this spectral region. Despite best efforts with normalization, this can cause artificial features and large errors in relative intensities. It can therefore not be excluded that the relative intensities and, thus, the angular dependence is affected by artifacts in the normalization procedure. Moreover, that the signal for $50 \%$ saturation is significantly smaller than for $100 \%$ (shown in Fig. 8b).

Alternatively, this result could suggest that the structure is a mixture of flat and upright geometries contributing differently to the spectra of the two different edges. Indeed, adding between $25-35 \%$ of upright monomers to the system does not alter the character of the $\mathrm{N}$ K-edge, however it reverses the angular dependence of the $\pi^{*}$-resonances in the $\mathrm{C}$ K-edge as shown in Fig. 6b. We can therefore conclude that low coverage BTA chains are dominant in the low coverage adsorption of benzotriazole on $\mathrm{Cu}(111)$, however 25-35\% of upright monomers might also be present.

\section{Structure at high coverage}

The structure of benzotriazole deposited at $100 \%$ saturation on $\mathrm{Cu}(111)$ was studied with NEXAFS, both for the $\mathrm{N}$ and the $\mathrm{C}$ K-edge, and NIXSW. While NEXAFS is capable of determining bond angles, NIXSW allows the determination of the height of atoms above 
a single crystal surface. Therefore the combination of the two techniques provides a more complete picture of the system.

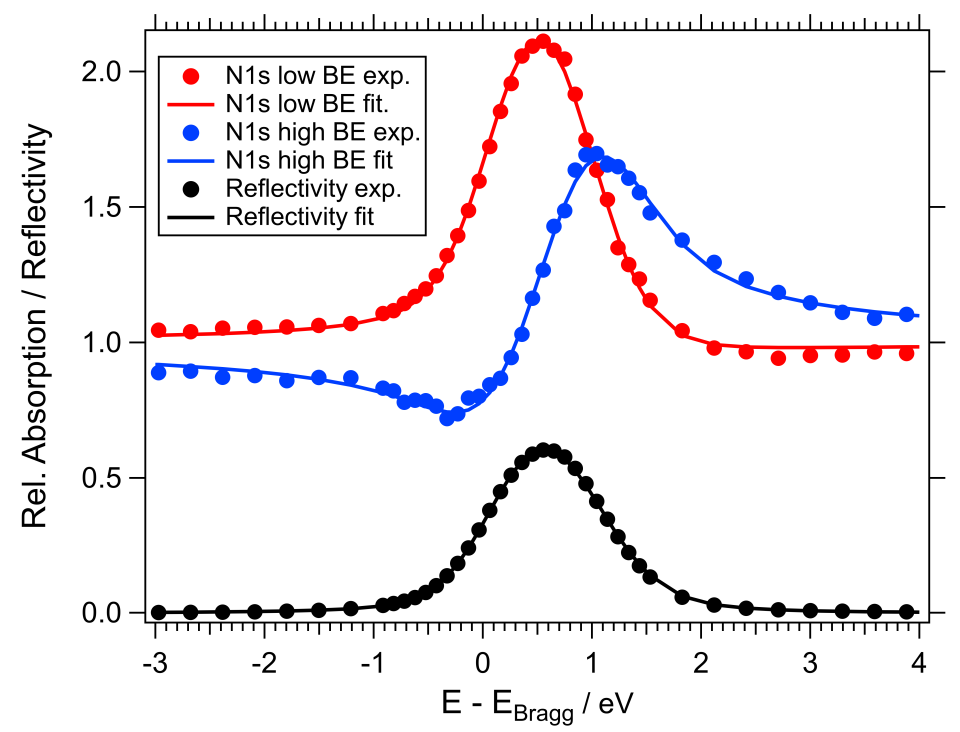

Figure 7: Experimental data and fits to the NIXSW Bragg reflection (black curve) and peak intensity curves of the two resolved $\mathrm{N}$ 1s peaks (red: low BE signal; blue high BE signal).

NIXSW data were acquired for the saturated chemisorbed BTA layer dosed at $320 \mathrm{~K}$ (for $9 \mathrm{~min})$. A detailed description of the data analysis and representative experimental data are included in the Supporting Information. All DFT models predict a wide distribution of heights of carbon atoms within the aromatic rings, which are all contributing to the single C 1s peak at $284.8 \mathrm{eV}$. Therefore, these positions cannot be determined by NIXSW. The number of nitrogen atoms is smaller, their height distribution narrower, and there is a clear shift in binding energy between different species, which makes the determination of their positions far more viable. Figure 7 shows the energy dependence of the low and high BE $\mathrm{N}$ 1s peaks (399.4 and $400.3 \mathrm{eV}$ ) and the reflectivity curve together with fits. The coherent fractions, $f_{\text {coh }}$, and positions, $p_{\text {coh }}$, resulting from the fits (and calculated using Eq. 1) are listed in Table 1 together with values expected for different BTA model geometries using the coordinates from the DFT-optimized structures in Fig. 2. The fit to the absorption curve of the low BE peak yields a very low coherent fraction of 0.28 , which is indicative of either disorder or atoms in different positions contributing to this peak. Typical values for atoms 
Table 1: Coherent fractions, $f_{\text {coh }}$, and positions, $p_{\text {coh }}$ (in units of $d_{111}$ ), determined from the fits to the NIXSW absorption curves for the low and high BE N 1s peaks and values expected for different models, as described in the text. The error bars of the experimental data are determined from the variance between different experiments

\begin{tabular}{lcccc}
\hline & Low BE peak $(399.4 \mathrm{eV})$ & \multicolumn{2}{c}{ High BE peak $(400.3 \mathrm{eV})$} \\
& $f_{\text {coh }}$ & $p_{\text {coh }}$ & $f_{\text {coh }}$ & $p_{\text {coh }}$ \\
\hline Experiment & $0.28 \pm 0.03$ & $0.36 \pm 0.01$ & $0.68 \pm 0.02$ & $0.95 \pm 0.01$ \\
\hline Single geometry models & & & & \\
BTA Monomer & 0.99 & 0.37 & 1.00 & 0.01 \\
BTA Dimers & 0.29 & 0.16 & 0.99 & 0.89 \\
BTA LC Chains (LCC) & 0.99 & 0.37 & 0.41 & 0.17 \\
BTA HC Chains (HCC) & 0.94 & 0.39 & 0.98 & 0.07 \\
Mixed geometry models & & & & \\
20\% LCC + 80\% Dimers & 0.34 & 0.26 & 0.78 & 0.91 \\
30\% LCC + 70\% Dimers & 0.39 & 0.28 & 0.69 & 0.92 \\
35\% LCC + 65\% Dimers & 0.43 & 0.30 & 0.63 & 0.93 \\
40\% LCC + 60\% Dimers & 0.47 & 0.31 & 0.59 & 0.93 \\
$50 \%$ LCC + 50\% Dimers & 0.55 & 0.33 & 0.50 & 0.96 \\
\hline
\end{tabular}

at a single position are around 0.9. A comparison with the values expected for the DFT model geometries shows that only the BTA dimers model leads to a similar coherent fraction for this peak, however the coherent positions and $f_{\text {coh }}$ for the high BE peaks do not match as well. Better agreement can be achieved by mixing dimer and chain structures and the results for mixed BTA low coverage chains and BTA dimers geometries are listed in the lower part of Table 1. Satisfying agreement with the experimental data is achieved for mixtures between $30 \%: 70 \%$ and $35 \%: 65 \%$. It should be noted that the coherent fraction can be significantly affected by small levels of contamination and beam damage, which cannot be completely ruled out in these experiments. Therefore one expects a larger discrepancy between experiment and model in these values. The experimental error bars stated in Table 1 are based to the variance of the fits to different data sets and do not include such systematic errors.

Experimental NEXAFS spectra for the $\mathrm{N}$ and C K-edge were obtained for $\theta=17^{\circ}, 42^{\circ}$, $67^{\circ}, 87^{\circ}$ and they are shown in Fig. 8 as solid lines. The dashed lines are the best theoretical fit after comparison with the structures in Fig. 2 and the NIXSW findings. The theoretical 
a) N K-edge

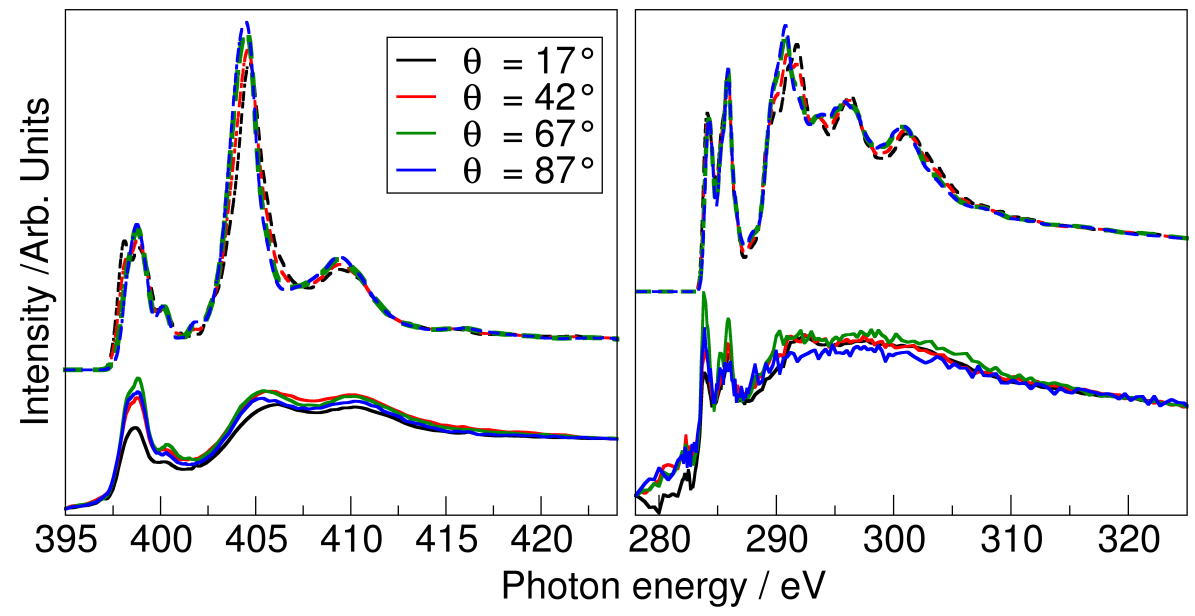

Figure 8: Comparison of experimental and computational spectra for the a) N K-edge and b) C K-edge for high coverage BTA adsorption at $300 \mathrm{~K}$. Experimental data are shown as solid lines. The computational spectra (dashed lines) are those for a mixture of $30 \%$ low coverage chains (Fig. 2b) and $70 \%$ dimers (Fig. 2c). The angle $\theta$ refers to the angle between the X-ray polarization vector and the surface normal (see Fig. 1).

spectra which are shown correspond to the $30 \%$ low coverage chains and $70 \%$ dimers mixture which is the best fit with the NIXSW data. Good agreement between this structure and the experimental data is obtained. For the N K-edge (Fig. 8a) the experimental data show two distinct $\pi^{*}$-resonance peaks and broad $\sigma^{*}$ features $\sim 5 \mathrm{eV}$ apart. While the position of the $\pi^{*}$ peaks is the same for all angles, $398.8 \mathrm{eV}$ and $400.3 \mathrm{eV}$, a small shift is observed in the positions of the $\sigma^{*}$ peaks, from $406.1 \mathrm{eV}$ for small incidence angles $\left(\theta=17^{\circ}\right)$ to 405.3 $\mathrm{eV}$ at high incidence angles $\left(\theta=87^{\circ}\right)$. It is worth noting that the angle dependence of the $\pi^{*}$ and $\sigma^{*}$-resonance peaks is also in good agreement with the computed spectra of the high coverage chains (see Fig. 2d and Fig. S2d). Therefore, without the extra information provided by NIXSW, the spectra could instead be assigned to this very different structure. We can thus conclude that the high coverage structure of benzotriazole on $\mathrm{Cu}(111)$ consist of dissociated BTA mostly arranged in dimers and partially as chains of alternating upright 
and flat molecules.

\section{Adatoms on the $\mathrm{Cu}(111)$ surface}

As the chemical shifts in XPS strongly depend on the coordination of the emitter atoms, one would expect a significant shift in the binding energy of the $\mathrm{Cu} 2 \mathrm{p}$ photo-electrons emitted from the $\mathrm{Cu}$ adatoms with respect to the bulk signal. To our knowledge, the only experimental value available for a $\mathrm{Cu} 2 \mathrm{p}_{3 / 2}$ surface core level shift is that of $\mathrm{Cu}$ layers of variable thickness on a $\mathrm{Ni}(111)$ surface, $\Delta \mathrm{E}_{\mathrm{B}}^{\exp }=-0.31 \mathrm{eV} \cdot{ }^{53}$

Table 2: Calculated chemical shifts, $\Delta \mathrm{E}_{\mathrm{B}}$, of the $\mathrm{Cu} 2 \mathrm{p}_{3 / 2}$ levels for surface atoms and copper adatoms $\left(\mathrm{Cu}_{\mathrm{ad}}\right)$ with respect to the bulk $\left(\Delta \mathrm{E}_{\mathrm{B}}=0 \mathrm{eV}\right)$. All values are in $\mathrm{eV}$. Two copper adatoms are present in the unit cell of the chains, and only one $\mathrm{Cu}_{\mathrm{ad}}$ for the dimers.

\begin{tabular}{lccc}
\hline Structure & \multicolumn{2}{c}{$\mathrm{Cu}_{\mathrm{ad}}$} & Surface \\
\hline BTA LC Chains & -0.50 & -0.43 & -0.21 \\
BTA Dimers & +0.60 & - & -0.17 \\
BTA HC Chains & -0.34 & -0.53 & -0.25 \\
\hline $\mathrm{Cu}$ slab flat & - & - & -0.28 \\
$\mathrm{Cu}$ slab w. adatoms & -0.71 & -0.70 & -0.28 \\
\hline
\end{tabular}

Our calculations for the surface layer of a 7-layer thick $\mathrm{Cu}(111)$ slab yield a surface core level shift of $\Delta \mathrm{E}_{\mathrm{B}}=-0.28 \mathrm{eV}$ with respect to the bulk atoms, which is in very good agreement with the experimental value. ${ }^{53}$ Copper adatoms on the same slab (with no other adsorbates) lead to $\Delta \mathrm{E}_{\mathrm{B}} \sim-0.71 \mathrm{eV}$ (see Table 2, bottom). When BTA molecules are present on the surface, the binding energy shifts for the adatoms are reduced, or can even change sign, depending on the structure (see Table 2 , top), leading to values between $\Delta \mathrm{E}_{\mathrm{B}}=$ $-0.53 \mathrm{eV}$ and $\Delta \mathrm{E}_{\mathrm{B}}=0.60 \mathrm{eV}$. This indicates different levels of charge transfer through the $\mathrm{N}-\mathrm{Cu}_{\mathrm{ad}}$ bonds, which appears strongest for the dimers $\left(\Delta \mathrm{E}_{\mathrm{B}}=0.60 \mathrm{eV}\right)$. The calculated core level shifts of the $\mathrm{Cu}$ atoms within the surface layer deviate very little from the flat clean $\mathrm{Cu}(111)$ surface, with values between -0.25 and $-0.17 \mathrm{eV}$.

Model spectra were calculated by a weighed superposition of Gaussian-Lorentzian peaks (G/L mixing $90 \%$; FWHM $0.8 \mathrm{eV}$, as determined from the experimental data), shifted by the 


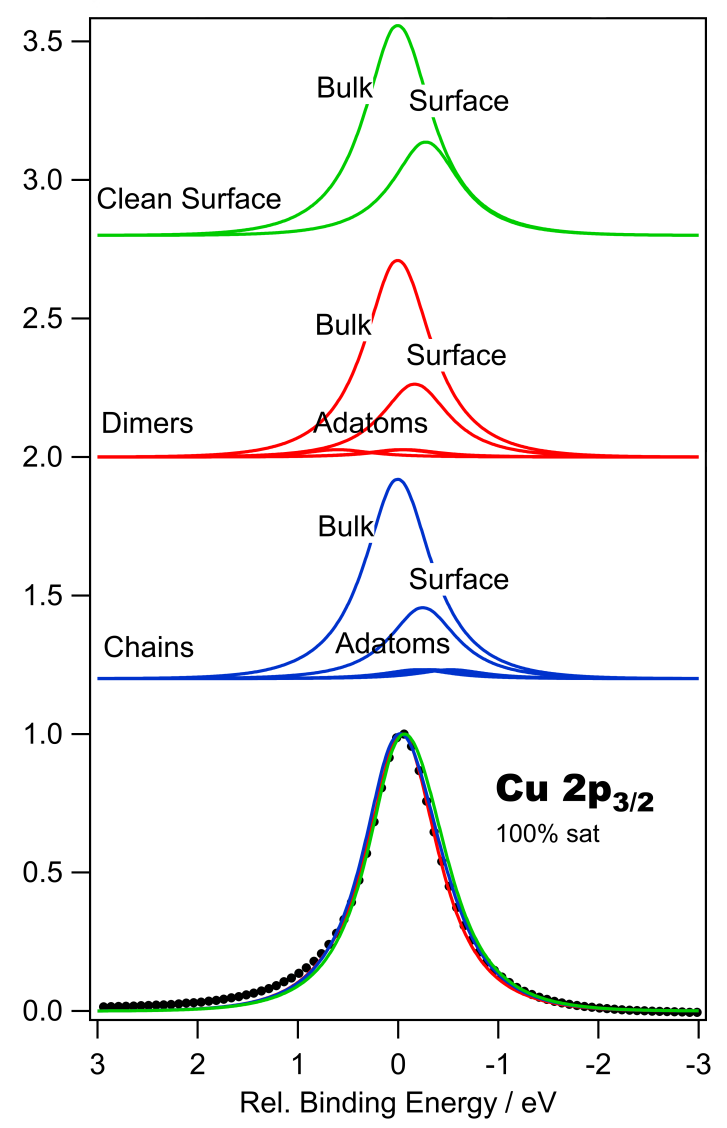

Figure 9: Comparison of experimental (black dots) and model $\mathrm{Cu} 2 \mathrm{p}_{3} / 2$ spectra for the two high coverage systems of deprotonated BTA with high density of $\mathrm{Cu}_{\mathrm{ad}}$, high-coverage chains (blue lines), high-coverage domains (red lines), and the clean $\mathrm{Cu}(111)$ surface without adatoms (green lines). The peaks representing the individual contributions to the spectra are plotted in the upper part of the Figure, the combined model spectra (superposition of all individual peaks) is plotted together with the experimental data.

respective values for the adatoms, surface layer and first bulk layers (assuming an inelastic mean free path of $5.6 \AA^{50}$ ). The comparison of model spectra for different models shows that the signal from the adatoms becomes virtually indiscernible from the spectrum expected for the clean surface (see Fig. 9) due to the low concentration of copper adatoms (the $\mathrm{Cu}_{\mathrm{ad}} / \mathrm{Cu}_{\text {slab }}$ ratio is $1 / 6$ at its most dense). Within the experimental error and - more importantly - due to the uncertainties imposed by intensity variations caused by photoelectron diffraction, it is, therefore, not possible to confirm the presence of $\mathrm{Cu}$ adatoms, nor their absence, in this adsorption structure by comparing experimental and model spectra. 


\section{Discussion}

In the present work we have shown that a combination of spectroscopic techniques and DFT calculations provides a detailed description of the adsorption of a complex organic molecule on a substrate. In particular, we have studied benzotriazole on $\mathrm{Cu}(111)$ for a wide range of temperatures and at two coverages, providing new insight into its adsorption behavior. The benzotriazole/Cu system has long been studied; a variety of structures have been reported in the literature, at seemingly similar conditions, thus prompting the need for further work to improve our understanding of this system and to bring clarity to previous results. In the present work we used a combination of synchrotron-based spectroscopic experimental techniques (NEXAFS, XPS, TP-XPS, NIXSW) and DFT.

We shed a light on the structure of the adsorbed film, of the substrate and on the temperature evolution of the system. We have identified that up to $280 \mathrm{~K}$ benzotriazole forms adsorbed layers of intact $\mathrm{BTAH}$ on $\mathrm{Cu}(111)$. At room temperature the molecule is dissociated into BTA and $\mathrm{H}$, confirming the results of previous studies at room temperature in UHV. ${ }^{25-27,54}$ Previous computational work has also shown that deprotonated adsorbed systems of benzotriazole are more stable ${ }^{16,19}$ and that the energy barrier for deprotonation is relatively low at below $1 \mathrm{eV} .{ }^{55}$

Both at low and high coverage, complex structures with coexisting different molecular arrangements are observed. At low coverage, the predominant structure is chains of alternating upright and flat BTAs (Fig. 2b). An excess of $\sim 20 \%$ of upright molecules with respect to flat-lying ones might also be present and they might appear either at the end of the low coverage chains or interspersed between the chains. At high coverage, a structure composed primarily of highly packed dimers is observed, and around $30 \%$ of low coverage chains coexist. A combination of techniques was found necessary to identify these structures unambiguously. XPS, which was used to probe the local chemical environment of the $\mathrm{N}$ atoms, gave theoretical spectra with relatively small differences between the different structures considered. It was thus sufficient to determine the protonation state of the molecule, 
but not the exact overlayer structure. The latter was obtained through NEXAFS, probing the orientation of molecular orbitals and NIXSW, providing information on the moleculesurface bond lengths. Both were needed to correctly identify the high coverage structures as predominantly dimers rather than high coverage chains.

The copper adatoms, which are vital to stabilize these chain and dimer structures, are not detected via XPS. We have shown that despite significant chemical shifts of around 0.5 $\mathrm{eV}$ the low $\mathrm{Cu}_{\mathrm{ad}}$ concentration renders their contribution to the overall $\mathrm{Cu} 2 \mathrm{p}$ XPS signal insignificant. However, having established that benzotriazole is dissociated (through the comparison of experimental and theoretical XPS and NEXAFS spectra), their presence can be inferred by previous DFT work which has shown ${ }^{16}$ (see also Fig. S6) that the adsorption of BTA on $\mathrm{Cu}(111)$ is only favorable when it is aided by $\mathrm{Cu}$ adatoms.

Previous experimental studies of high coverage benzotriazole in UHV on $\mathrm{Cu}(111)$ have also shown a mostly upright adsorption of the molecules through the azole moiety, however they differ from each other and the present study in the predicted overlayer structure. Fang et $a l .{ }^{24}$ predicted a structure of upright chains connected by $\mathrm{H}$-bonds between neighboring benzene and azole rings. The H-bond is however supposed to be very weak in this configuration $^{56}$ and the structure itself has subsequently been contested. ${ }^{54}$ The newly proposed structure in Ref. ${ }^{54}$ is instead of almost upright BTAs connected by copper adatoms, possibly forming chains. NEXAFS results by Walsh et al. ${ }^{25}$ also suggested an almost upright adsorption of (deprotonated) single upright BTAs on the $\mathrm{Cu}(100)$ surface. The data from these studies and from the present work do agree with the orientation of high coverage BTA, i.e. almost upright. These previous results can be re-read in light of the present findings and a good agreement across the board is obtained. In fact, the NEXAFS spectra of Ref. ${ }^{25}$ are very close to those obtained in the present study, and thus could be re-interpreted in the same way. In Ref., ${ }^{54}$ XPS and UPS show that deprotonated BTA adsorbs via the triazole moiety and that the orientation of the molecule is slightly tilted with respect to the surface normal. Similarly, Ref. ${ }^{24}$ reports deprotonation, the plane of the molecule tilted from the 
surface and possible BTA-Cu polymerization. Again, these results are consistent with the present findings.

Finally, the dimer structures found to be prevalent in our study have also been observed by Grillo et al. ${ }^{26,27}$ by means of scanning tunneling microscopy (STM). A significant difference, however, between the present work and Ref. ${ }^{26}$ is the prevalence of the dimers also at low coverage, rather than chains. This could be due to differences in the surface condition. Fig. S1 shows that the conditions of the surface can affect which $\mathrm{Cu}_{\mathrm{ad}}$-aided structures are favorable, dimers being expected when the energy cost of forming the adatoms is very high. It could thus be possible that this set of experiments fall into this range especially since the surface in Ref. ${ }^{26}$ is reconstructed. Despite it being quite unusual, adsorbate-induced reconstructions have been observed for $\mathrm{Cu}(111) .{ }^{57-60}$ While the cause for the reconstruction is unknown in this case, its presence could have two important consequences: it could make the surface atoms less mobile, thus disfavoring the presence of adatoms at the sites needed to form chains, and it could provide templating effects which again could favor dimers over chains.

The present work also showed that the compact, high coverage layer of dissociated BTA is stable up to at least $450 \mathrm{~K}$. Since dissociated BTA is strongly adsorbed to the surface, ${ }^{16,18-21}$ this molecular "barrier" can perform a protective action towards $\mathrm{Cu}(111)$ via a competitive adsorption mechanism. ${ }^{7}$ The protective action of the BTA overlayer against corrosion can be expected up to $670 \mathrm{~K}$ when complete desorption or decomposition is observed. The layer, however, becomes steadily less stable from $430 \mathrm{~K}$, when desorption and decomposition start being appreciable, and thus corrosion inhibition is expected to decrease in this temperature range. This is in good agreement with previous findings of benzotriazole reducing oxidation of copper up to $\sim 620 \mathrm{~K} \cdot{ }^{10,52}$ 


\section{Conclusions}

The present work demonstrates that the synergy between several spectroscopic techniques and DFT calculations is necessary to reveal complex details of this system of organic molecules on a substrate. Indeed, DFT has helped clarify seemingly contradictory N and C NEXAFS spectra for low coverage adsorption, showing them to be compatible with an excess of upright molecules in the system, which is however dominated by chains. Similarly, the combinations of NEXAFS, NIXSW and DFT leads to the identification of a dimer/chain mixture as the high coverage structure, rather than the theoretically more energetically stable chains. This combination of techniques has also been pivotal in clarifying the experimental finding of a seemingly "clean" surface, i.e. without copper adatoms, observed with XPS. Indeed, DFT has shown that a distinct surface feature such as copper adatoms, resulting in theoretical XPS shifts on the order of $\sim 0.5 \mathrm{eV}$, does not affect the overall XPS spectra at the concentration needed in the adsorbed systems.

\section{Acknowledgement}

A.M.s work is partly supported by the European Research Council under the European Unions Seventh Framework Programme (FP/20072013)/ERC Grant Agreement no. 616121 (HeteroIce project). The authors are grateful for computational resources to UCL research computing, the U.K. Car-Parrinello Consortium UKCP (EP/F036884/1), for access to Archer, and the Materials and Molecular Modelling Hub, which is partly funded by EPSRC (EP/P020194/1). The calculation of the theoretical NEXAFS spectra used resources of the National Energy Research Scientific Computing Center (NERSC), a U.S. Department of Energy Office of Science User Facility operated under Contract No. DE-AC02-05CH11231. C.G. performed part of this work as a user project at the Molecular Foundry which is supported by the Office of Science, Office of Basic Energy Sciences, of the U.S. Department of Energy under Contract No. DE-AC02-05CH11231. Diamond Light source is acknowledged 
for access to beamline I09, technical and travel support under beamtime award SI12800. The authors particularly acknowledge the help of Tien-Lin Lee, Pardeep Kumar Thakur and David McCue during the experiments. P.T acknowledges part-funding for a PhD studentship from Diamond Light Source.

\section{Supporting Information Available}

Theoretical NEXAFS and XPS spectra and formation energies for all the BTAH and BTA adsorption models are presented. Description of the NIXSW data analysis procedure and sample data.

\section{References}

(1) Kuznetsov, Y. Organic Inhibitors of Corrosion of Metals; Springer US: New York, 1996.

(2) Spikes, H. Friction Modifier Additives. Tribol. Lett. 2015, 60, 5.

(3) Nazeeruddin, M. K.; Baranoff, E.; Grtzel, M. Dye-sensitized solar cells: A brief overview. Sol. Energy 2011, 85, $1172-1178$.

(4) Howes, P. D.; Rana, S.; Stevens, M. M. Plasmonic nanomaterials for biodiagnostics. Chem. Soc. Rev. 2014, 43, 3835-3853.

(5) Gattinoni, C.; Michaelides, A. Atomistic details of oxide surfaces and surface oxidation: the example of copper and its oxides. Surf. Sci. Rep. 2015, 70, 424-447.

(6) Procter \& Gamble, Ltd., Compositions for Inhibiting Metal Tarnish. 1947.

(7) Finsgar, M.; Milosev, I. Inhibition of copper corrosion by 1,2,3-benzotriazole: A review. Corr. Sc. 2010, 52, 2737-2749. 
(8) Dugdale, I.; Cotton, J. B. An electrochemical investigation on the prevention of staining of copper by benzotriazole. Corros. Sci. 1963, 3, 69-74.

(9) Cotton, J. B.; Scholes, I. R. Benzotriazole and Related Compounds as Corrosion Inhibitors For Copper. Brit. Corros. J. 1967, 2, 1-5.

(10) Poling, G. Reflection infra-red studies of films formed by benzotriazole on Cu. Corr. Sci. 1970, 10, 359-370.

(11) Nefedov, A.; Wöll, C. In Surface Science Techniques; Bracco, G., Holst, B., Eds.; Springer Berlin Heidelberg: Berlin, Heidelberg, 2013; p 277.

(12) Kokalj, A.; Peljhan, S. Density Functional Theory Study of ATA, BTAH, and BTAOH as Copper Corrosion Inhibitors: Adsorption onto $\mathrm{Cu}(111)$ from Gas Phase. Langmuir 2010, 26, 14582-14593.

(13) Kokalj, A.; Kovačević, N.; Peljhan, S.; Finšgar, M.; Lesar, A.; Milošev, I. Triazole, Benzotriazole, and Naphthotriazole as Copper Corrosion Inhibitors: I. Molecular Electronic and Adsorption Properties. ChemPhysChem 2011, 12, 3547-3555.

(14) Peljhan, S.; Kokalj, A. DFT study of gas-phase adsorption of benzotriazole on Cu(111), $\mathrm{Cu}(100), \mathrm{Cu}(110)$, and low coordinated defects thereon. Phys. Chem. Chem. Phys. 2011, 13, 20408-20417.

(15) Jiang, Y.; Adams, J. B. First principle calculations of benzotriazole adsorption onto clean $\mathrm{Cu}(111)$. Surf. Sci. 2003, 529, $428-442$.

(16) Gattinoni, C.; Michaelides, A. Understanding corrosion inhibition with van der Waals DFT methods: the case of benzotriazole. Faraday Discuss. 2015, 180, 439-458.

(17) Finšgar, M.; Lesar, A.; Kokalj, A.; Milošev, I. A comparative electrochemical and quantum chemical calculation study of BTAH and BTAOH as copper corrosion inhibitors in near neutral chloride solution. Electrochim. Acta 2008, 53, 8287-8297. 
(18) Chen, X.; Hakkinen, H. Divide and Protect: Passivating $\mathrm{Cu}(111)$ by $\mathrm{Cu}^{-}$ (benzotriazole) 2 . J. Phys. Chem. C 2012, 116, 22346-22349.

(19) Kokalj, A.; Peljhan, S.; Finsgar, M.; Milosev, I. What Determines the Inhibition Effectiveness of ATA, BTAH, and BTAOH Corrosion Inhibitors on Copper? J. Am. Chem. Soc. 2010, 132, 16657-16668.

(20) Kokalj, A. Ab initio modeling of the bonding of benzotriazole corrosion inhibitor to reduced and oxidized copper surfaces. Faraday Discuss. 2015, 180, 415-438.

(21) Peljhan, S.; Koller, J.; Kokalj, A. The Effect of Surface Geometry of Copper on Adsorption of Benzotriazole and Cl. Part I. J. Phys. Chem. C 2014, 118, 933-943.

(22) Roberts, R. F. X-Ray photoelectron spectroscopic characterization of copper oxide surfaces treated with benzotriazole. J. Electron Spectrosc. Relat. Phenom. 1974, 4, 273291.

(23) Rubim, J. C.; Gutz, I. G. R.; Sala, O.; Orvillethomas, W. J. Surface enhanced Ramanspectra of benzotriazole adsorbed on a copper electrode. J. Mol. Struct. 1983, 100, $571-583$.

(24) Fang, B. S.; Olson, C. G.; Lynch, D. W. A photoemission-study of benzotriazole on clean copper and cuprous-oxide. Surf. Sci. 1986, 176, 476-490.

(25) Walsh, J. F.; Dhariwal, H. S.; Gutierrez-Sosa, A.; Finetti, P.; Muryn, C. A.; Brookes, N. B.; Oldman, R. J.; Thornton, G. Probing molecular orientation in corrosion inhibition via a NEXAFS study of benzotriazole and related molecules on $\mathrm{Cu}(100)$. Surf. Sci. 1998, 415, 423-432.

(26) Grillo, F.; Tee, D. W.; Francis, S. M.; Fruechtl, H.; Richardson, V., N Initial stages of benzotriazole adsorption on the $\mathrm{Cu}(111)$ surface. Nanoscale 2013, 5, 5269-5273. 
(27) Grillo, F.; Tee, D. W.; Francis, S. M.; Fruechtl, H. A.; Richardson, N. V. Passivation of Copper: Benzotriazole Films on $\mathrm{Cu}(111)$. J. Phys. Chem. C 2014, 118, 8667-8675.

(28) Velasco-Velez, J.-J.; Wu, C. H.; Pascal, T. A.; Wan, L. F.; Guo, J.; Prendergast, D.; Salmeron, M. The structure of interfacial water on gold electrodes studied by x-ray absorption spectroscopy. Science 2014, 346, 831-834.

(29) Ontaneda, J.; Nicklin, R. E. J.; Cornish, A.; Roldan, A.; Grau-Crespo, R.; Held, G. Adsorption of Methyl Acetoacetate at Ni111: Experiment and Theory. J. Phys. Chem. C 2016, 120, 27490-27499.

(30) Tsaousis, P.; Ontaneda, J.; Bignardi, L.; Bennett, R. A.; Grau-Crespo, R.; Held, G. Combined Experimental and Theoretical Study of Methyl Acetoacetate Adsorption on Ni100. J. Phys. Chem. C 2018, 122, 6186-6194.

(31) Fronzoni, G.; Balducci, G.; De Francesco, R.; Romeo, M.; Stener, M. Density Functional Theory Simulation of NEXAFS Spectra of Molecules Adsorbed on Surfaces: $\mathrm{C}_{2} \mathrm{H}_{4}$ on Si(100) Case Study. J. Phys. Chem. C 2012, 116, 18910-18919.

(32) Siefermann, K. R. et al. Atomic-Scale Perspective of Ultrafast Charge Transfer at a Dye-Semiconductor Interface. J. Phys. Chem. Lett. 2014, 5, 2753-2759.

(33) Woodruff, D. P. Surface structure determination using x-ray standing waves. Rep. Prog. Phys. 2005, 68, 743.

(34) Fisher, C. J.; Ithin, R.; Jones, R. G.; Jackson, G. J.; Woodruff, D. P.; Cowie, B. C. C. Non-dipole photoemission effects in x-ray standing wavefield determination of surface structure. J. Phys. Condens. Matter 1998, 10, L623-L629.

(35) Kresse, G.; Hafner, J. Ab initio molecular dynamics for liquid metals. Phys. Rev. B $1993,47,558-561$. 
(36) Kresse, G.; Hafner, J. Ab initio molecular-dynamics simulation of the liquidmetalamorphous-semiconductor transition in germanium. Phys. Rev. B 1994, 49, $14251-14269$.

(37) Kresse, G.; Furthmueller, J. Efficiency of ab-initio total energy calculations for metals and semiconductors using a plane-wave basis set. Comp. Mat. Sci. 1996, 6, 15-50.

(38) Kresse, G.; Furthmueller, J. Efficient iterative schemes for ab initio total-energy calculations using a plane-wave basis set. Phys. Rev. B 1996, 54, 11169-11186.

(39) Klimes, J.; Bowler, D. R.; Michaelides, A. Van der Waals density functionals applied to solids. Phys. Rev. B 2011, 83, 195131.

(40) Kresse, G.; Joubert, D. From ultrasoft pseudopotentials to the projector augmentedwave method. Phys. Rev. B 1999, 59, 1758-1775.

(41) Kohler, L.; Kresse, G.; Schmid, M.; Lundgren, E.; Gustafson, J.; Mikkelsen, A.; Borg, M.; Yuhara, J.; Andersen, J. N.; Marsman, M.; Varga, P. High-coverage oxygen structures on $\operatorname{Rh}(111)$ : Adsorbate repulsion and site preference is not enough. Phys. Rev. Lett. 2004, 93, 266103.

(42) Prendergast, D.; Galli, G. X-ray absorption spectra of water from first principles calculations. Phys. Rev. Lett. 2006, 96, 215502.

(43) Giannozzi, P. et al. QUANTUM ESPRESSO: a modular and open-source software project for quantum simulations of materials. J. Phys. Cond. Matt. 2009, 21, 395502.

(44) Taillefumier, M.; Cabaret, D.; Flank, A.-M.; Mauri, F. X-ray absorption near-edge structure calculations with the pseudopotentials: Application to the $K$ edge in diamond and $\alpha$-quartz. Phys. Rev. B 2002, 66, 195107. 
(45) Prendergast, D.; Louie, S. G. Bloch-state-based interpolation: An efficient generalization of the Shirley approach to interpolating electronic structure. Phys. Rev. B 2009, $80,235126$.

(46) Shirley, E. L. Optimal basis sets for detailed Brillouin-zone integrations. Phys. Rev. B 1996, 54, 16464-16469.

(47) Perdew, J. P.; Burke, K.; Ernzerhof, M. Generalized gradient approximation made simple. Phys. Rev. Lett. 1996, 77, 3865-3868.

(48) Vanderbilt, D. Soft Self-consistent pseudopotentials in a generalized eigenvalue formalism. Phys. Rev. B 1990, 41, 7892-7895.

(49) Stöhr, J. NEXAFS Spectroscopy; Springer Series in Surface Sciences; Springer-Verlag Berlin Heidelberg, 1992.

(50) Tanuma, S.; Powell, C. J.; Penn, D. R. Calculations of electron inelastic mean free paths. Surf. Interface Anal. 1991, 17, 911-926.

(51) Katritzky, A. R.; Wang, Z.; Tsikolia, M.; Hall, C. D.; Carman, M. Benzotriazole is thermally more stable than 1,2,3-triazole. Tetrahedron Lett. 2006, 47, 7653-7654.

(52) Walker, R. Benzotriazole as a corrosion ihibitor. Metal finishing 1973, 71, 63-66.

(53) Koschel, H.; Held, G.; Steinruck, H. P. The growth of thin Cu layers on Ni(111) studied by CO titration and photoelectron spectroscopy. Surf. Sci. 2000, 453, 201-213.

(54) Nilsson, J.-O.; Törnkvist, C.; Liedberg, B. Photoelectron and infrared reflection absorption spectroscopy of benzotriazole adsorbed on copper and cuprous oxide surfaces. Appl. Surf. Sci. 1989, 37, 306-326.

(55) Kokalj, A.; Peljhan, S.; Koller, J. The Effect of Surface Geometry of Copper on Dehydrogenation of Benzotriazole. Part II. J. Phys. Chem. C 2014, 118, 944-954. 
(56) Bougeard, D.; Le Calvé, N.; Saint Roch, B.; Novak, A. 1,2,4-Triazole: Vibrational spectra, normal coordinate calculations, and hydrogen bonding. J. Chem. Phys. 1976, $64,5152-5164$.

(57) Liu, D.-J.; Walen, H.; Oh, J.; Lim, H.; Evans, J. W.; Kim, Y.; Thiel, P. A. Search for the structure of a sulfur-induced reconstruction on $\mathrm{Cu}(111)$. J. Phys. Chem. C 2014, 118, 29218-29223.

(58) Muñoz-Márquez, M.; Parkinson, G.; Quinn, P.; Gladys, M.; Tanner, R.; Woodruff, D.; Noakes, T.; Bailey, P. N-induced pseudo-(100) reconstruction of $\mathrm{Cu}(111)$ : One layer or more? Surf. Sci. 2005, 582, 97-109.

(59) Moritani, K.; Okada, M.; Teraoka, Y.; Yoshigoe, A.; Kasai, T. Reconstruction of $\mathrm{Cu}(111)$ induced by a hyperthermal oxygen molecular beam. J. Phys. Chem. C 2008, 112, 8662-8667.

(60) Grönbeck, H. Thiolate induced reconstruction of $\mathrm{Au}(111)$ and $\mathrm{Cu}(111)$ investigated by density functional theory calculations. J. Phys. Chem. C 2010, 114, 15973-15978. 
Graphical TOC Entry

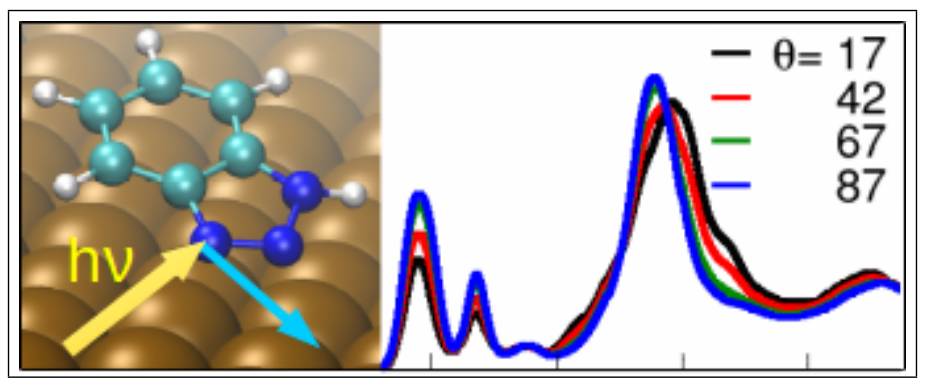

